FERMILAB-PUB-95/221-T

CLNS 95/1357

MRI-PHY/16/95

UIOWA-95-06

AZPH-TH-95-15

hep-ph/9512364

\title{
Ultrahigh-Energy Neutrino Interactions
}

\author{
Raj Gandhi ${ }^{1}$ \\ Mehta Research Institute \\ 10, Kasturba Gandhi Marg, Allahabad 211002, India \\ Chris Quigg $^{2}$ \\ Theoretical Physics Department, Fermi National Accelerator Laboratory \\ P.O. Box 500, Batavia, Illinois 60510 USA \\ and \\ Floyd R. Newman Laboratory of Nuclear Studies, Cornell University \\ Ithaca, New York 14853 USA \\ Mary Hall Reno ${ }^{3}$ \\ Department of Physics and Astronomy, University of Iowa \\ Iowa City, Iowa 52242 USA \\ Ina Sarcevic ${ }^{4}$ \\ Department of Physics, University of Arizona \\ Tucson, Arizona 85721 USA
}

\begin{abstract}
Cross sections for the interactions of ultrahigh-energy neutrinos with nucleons are evaluated in light of new information about nucleon structure functions. For $10^{20}-\mathrm{eV}$ neutrinos, the cross section is about 2.4 times previous estimates. We also review the cross sections for neutrino interactions with atomic electrons. Some consequences for interaction rates in the Earth and for event rates from generic astrophysical sources in large-scale detectors are noted.
\end{abstract}

Key words: Neutrino astronomy. Neutrino-nucleon scattering. Neutrino-electron scattering.

PACS: 13.15.+g, 13.60.Hb, 95.55.Vj, 96.40.Tv 


\section{Introduction}

Neutrino telescopes hold great promise for probing the deepest reaches of stars and galaxies [1-4]. As highly stable neutral particles, neutrinos arrive at a detector on a direct line from their source, undeflected by intervening magnetic fields. Whereas high-energy photons are completely absorbed by a few hundred grams $/ \mathrm{cm}^{2}$ of material, the interaction length of a $1-\mathrm{TeV}$ neutrino is about 250 kilotonnes $/ \mathrm{cm}^{2}$, which corresponds to a column of water 2.5 million kilometers deep. The feebleness of neutrino interactions means that neutrinos can bring us astrophysical information that other radiation cannot, but it also means that vast detectors are required to receive this information.

Encouragement to contemplate neutrino telescopes with effective volumes as large as $1 \mathrm{~km}^{3}$ comes from the observation of neutrinos correlated with supernova SN1987A [5] and from the detection of solar neutrinos not only by radiochemical methods [6-8] but also by observing the direction of recoil electrons from neutrino interactions [9]. At the same time, detection of neutrinos produced by cosmic-ray interactions in Earth's atmosphere $[10,11]$ has become commonplace in underground detectors [12] and has emerged as a tool for investigating neutrino oscillations [13-15].

A principal scientific goal of large-scale neutrino telescopes is the detection of ultrahigh-energy (UHE: $\gtrsim 10^{12} \mathrm{eV}$ ) cosmic neutrinos produced outside the atmosphere: neutrinos produced by galactic cosmic rays interacting with interstellar gas, and extragalactic neutrinos $[16,17]$. Extragalactic sources range from the conventional - the diffuse $\left(\sim 10^{18} \mathrm{eV}\right)$ neutrino flux produced by interactions over cosmological time of extragalactic cosmic rays with the microwave background radiation [18] - to the highly speculative - such as the diffuse flux associated with the decay of cosmic strings [19,20] and other topological defects [21] in the relatively late Universe .

Active galactic nuclei (AGNs) have long been considered as prodigious particle accelerators [22] and beam dumps [23], for they are the most powerful radiation sources known in the Universe, with typical luminosities in the range $10^{42}$ to $10^{48} \mathrm{erg} / \mathrm{s}$. These cosmic accelerators are presumably powered by the gravitational energy of matter spiraling in to a supermassive $\left(\sim 10^{8} M_{\odot}\right)$ black hole. Cosmic rays generated within an AGN may interact with matter or radiation in the AGN accretion disk, or with UV photons in the associated jets, to produce pions whose decay products include photons and neutrinos. The

\footnotetext{
1 Internet address: raj@mri.ernet.in

2 Internet address: quigg@fnal.gov

3 Internet address: reno@hephp1.physics.uiowa.edu

4 Internet address: ina@ccit.arizona.edu
} 
dominant mechanisms for photon and neutrino production are

$$
\begin{aligned}
p(p / \gamma) \rightarrow & \pi^{0}+\text { anything } \\
& \longrightarrow \gamma \gamma
\end{aligned}
$$

and

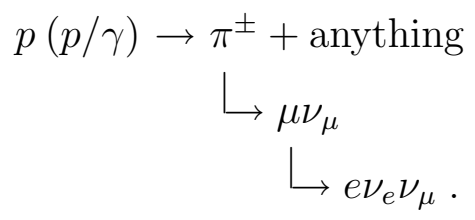

If $\pi^{+}, \pi^{-}$, and $\pi^{0}$ are produced in equal numbers, the relative populations of the neutral particles will be $2 \gamma: 2 \nu_{\mu}: 2 \bar{\nu}_{\mu}: 1 \nu_{e}: 1 \bar{\nu}_{e}$. Taken together, neutrino emission from ordinary AGNs may provide the dominant isotropic flux at energies above about $10^{4} \mathrm{GeV}$.

The recent detection of energetic photons $\left(E_{\gamma}>100 \mathrm{MeV}\right)$ from some 40 AGNs in the Energetic Gamma-Ray Experiment Telescope (EGRET) full-sky survey [24] may signal the existence of individual point-sources of neutrinos. EGRET, a multilevel thin-plate spark chamber device aboard the Compton Gamma-Ray Observatory, has also detected more than a dozen extragalactic sources at photon energies above $1 \mathrm{GeV}$. The EGRET sources have the characteristics of blazars, AGNs that have associated jets closely aligned with the observer's line of sight. The closest EGRET source is Markarian 421, a BL Lacertae object at redshift $z=0.031$. In 1992, Mrk 421 was detected in air showers as a source of $\mathrm{TeV}$ photons by the ground-based Whipple Observatory, an optical reflector with a 10-meter aperture viewed by more than 100 small phototubes [25]. In 1995, the Whipple Observatory Gamma-Ray Collaboration detected a second TeV photon source, Mrk 501, at $z=0.034$ [26]. If the $\mathrm{TeV}$ photons are products of $\pi^{0}$ decay, then these sources should also be copious neutrino emitters. If instead the $\mathrm{TeV}$ photons are produced by inverse Compton scattering of energetic electrons off ultraviolet photons, no UHE neutrinos will be created. The ability to observe UHE neutrinos from $\mathrm{TeV}$ photon sources would be an important new AGN diagnostic.

Ultrahigh-energy neutrinos can be detected by observing long-range muons produced in charged-current neutrino-nucleon interactions. To reduce the background from muons produced in the atmosphere, it is advantageous to site a neutrino telescope at a depth of several kilometers (water equivalent) or to observe upward-going muons. High neutrino energy brings a number of advantages. First, the charged-current cross section increases, as $\sigma \propto E_{\nu}$ for $E_{\nu} \lesssim 10^{12} \mathrm{eV}$, then as $\sigma \propto E_{\nu}^{0.4}$ for $E_{\nu} \gtrsim 10^{15} \mathrm{eV}$. Second, the background 
of atmospheric neutrinos falls away compared to the flux from extragalactic sources, approximately as $E_{\nu}^{-1.6}$. Cosmic neutrinos reflect the cosmic-ray spectrum near the source $\left(d N / d E \propto E^{-2}\right)$, whereas the atmospheric neutrino spectrum $\left(\propto E^{-3.6}\right.$ above $100 \mathrm{GeV}$ ) is about one power of the energy steeper than the cosmic-ray spectrum at the Earth $\left(\propto E^{-2.7}\right)$, which is steeper than the source spectrum [27]. The signal of interest for neutrino astronomy should emerge from the atmospheric-neutrino background at $E_{\nu} \sim 1-10 \mathrm{TeV}$. Third, the muon range grows with energy, increasing as $E_{\mu}$ for $E_{\mu} \lesssim 1 \mathrm{TeV}$, then increasing roughly as $\log E_{\mu}$ at higher energies. For upward-going muons, the effective volume of a neutrino telescope is thus equal to the instrumented area times the muon range.

Estimates of the fluxes of UHE neutrinos from AGNs and other astrophysical sources suggest that a surface area exceeding $0.1 \mathrm{~km}^{2}$ is required [28]. If the muons are detected by observing the Čerenkov light they produce when traversing a transparent medium of water or ice, huge target volumes are conceivable [29]. Four instruments specifically designed for high-energy neutrino detection are currently under construction: DUMAND [30,31], at a depth of $4760 \mathrm{~m}$ in the ocean $30 \mathrm{~km}$ off the island of Hawaii; the Baikal Neutrino Telescope [32], at a depth of $1 \mathrm{~km}$ in Lake Baikal in Siberia; NESTOR [33], $3500 \mathrm{~m}$ deep in the Mediterranean near Pylos, Greece; and AMANDA [34,31], in deep polar ice at the South Pole. All these detectors aim for effective areas of about $0.02 \mathrm{~km}^{2}$ and an angular resolution for TeV muons of approximately $1^{\circ}$. These detectors represent a giant step in instrumented volume from their underground predecessors. To reach an effective volume of $1 \mathrm{~km}^{3}$ will require efficiencies of scale for the water-Čerenkov technique or new means of detection. Radio detection is under active study [35]. Acoustic detection may become viable in the future [36].

At low neutrino energies $\left(E_{\nu} \ll M_{W}^{2} / 2 M\right.$, where $M_{W}$ is the intermediateboson mass and $M$ is the nucleon mass), differential and total cross sections for the reaction $\nu N \rightarrow \mu+$ anything are proportional to the neutrino energy. Above $E_{\nu} \approx 10^{12} \mathrm{eV}$, the gauge-boson propagator restricts the momentum transfer $Q^{2}$ to values near $M_{W}^{2}$ and damps the cross section. At ultrahigh energies, the $W$ propagator limits the effective interval in the fractional parton momentum $x$ to the region around $M_{W}^{2} / 2 M E_{\nu}$.

Since the UHE $\nu N$ cross sections were studied in detail nearly a decade ago [37-40], our knowledge of parton distributions has developed significantly. In place of parton distributions that were essentially based on a single data set [41], we now have at our disposal a number of sets of parton distributions derived from global fits to a rich universe of experimental information. At small values of $x$, parton distributions have been shaped by measurements made possible for the first time by the electron-proton collider HERA at DESY. The discovery of the top quark $[42,43]$ with a mass $m_{t} \approx 175 \mathrm{GeV} / c^{2}$ reduces 
the contribution of the $b$-quark sea to the neutrino-nucleon total cross section. This new information provides the incentive to reëxamine the cross sections for UHE $\nu N$ interactions [44].

In $\S 2$, we review what is known about the structure of the nucleon and explain how we treat the extrapolation to small values of $x$ that is crucial at the highest energies. Then in $\S 3$ we present in turn our calculations of the chargedcurrent and neutral-current cross sections, and explore the variations due to different sets of parton distributions. Although $\nu N$ interactions provide the dominant signal and account for most of the attenuation of neutrino beams in the Earth at high energies, the $W^{-}$resonance in the $\bar{\nu}_{e} e$ channel has a very strong effect for neutrino energies around 6.3 PeV. Accordingly, we review the interactions of neutrinos with electron targets in $\S 4$. Section 5 is devoted to a study of the attenuation of neutrinos in the Earth. We improve our treatment of this important effect by using a detailed model of the Earth's interior. We make some remarks about neutrino interactions in the atmosphere in $\S 6$, and comment in $\S 7$ on the possibility of observing the shadows of the Moon and Sun. In $\S 8$ we estimate the event rates from atmospheric neutrinos and from a variety of astrophysical sources in detectors with effective volumes of 0.1-1 $\mathrm{km}^{3}$. A final assessment concludes the paper.

We find that current knowledge of the proton's parton distributions allows us to calculate the $\nu N$ cross sections with confidence up to neutrino energies of about $10^{16} \mathrm{eV}$. The new cross sections are noticeably larger than those calculated a decade ago for energies above about $10^{15} \mathrm{eV}$. At $10^{20} \mathrm{eV}$, our nominal cross sections are about 2.4 times as large as those calculated using the EHLQ parton distributions $[37,38]$. At energies exceeding $10^{16} \mathrm{eV}$, our ignorance of proton structure at small values of $x$ is reflected in a spread of the cross sections calculated using various modern parton distributions. The resulting uncertainty reaches a factor of $2^{ \pm 1}$ at $10^{20} \mathrm{eV}$. The larger cross sections imply enhanced rates for downward-going muons produced in charged-current interactions. At the energies of interest for the observation of extraterrestrial neutrino sources, upward-going muon rates are little changed, because the increased reaction rate is compensated by increased attenuation of neutrinos traversing the Earth to reach the detector. We find that a detector with an effective area of $0.1 \mathrm{~km}^{2}$ and a muon energy threshold in the range of 1 to $10 \mathrm{TeV}$ should readily observe the diffuse flux of neutrinos expected from AGNs above the background of atmospheric neutrinos. The detection of cosmic neutrinos from the interaction of cosmic-ray protons with the microwave background appears a remote possibility, even for a $1-\mathrm{km}^{3}$ detector. 


\section{New Information about Nucleon Structure}

To compute the cross sections for neutrino-nucleon interactions at high energies, we require both a knowledge of the elementary matrix elements and also a detailed description of the quark structure of the nucleon. We have the first, thanks to extensive experimental validation of the $S U(2)_{L} \otimes U(1)_{Y}$ electroweak theory and refinement of the parameters that appear in the elementary neutrino-quark scattering. For the second, we rely on parton distribution functions extracted from studies of lepton-hadron scattering and of the productions of jets, intermediate bosons, dileptons, and photons in hadronhadron collisions. Systematic global fits to experimental data have greatly extended our knowledge of parton distribution functions and made modern parametrizations increasingly robust.

Although many experiments have nourished the steady improvement of the parton distributions, recent results from the ep collider HERA [45-49] are particularly informative for the application at hand. Measurements by the ZEUS and $\mathrm{H} 1$ collaborations mark the first experimental studies of very small parton momentum fractions $x$ at momentum transfers $Q^{2}$ securely in the deeply inelastic regime. The HERA experiments have begun to map the structure function $F_{2}\left(x, Q^{2}\right)$ in the interval $10^{-4} \lesssim x \lesssim 10^{-2}$, with $8.5 \mathrm{GeV}^{2} \lesssim Q^{2} \lesssim 15 \mathrm{GeV}^{2}$. For $x \gtrsim 2 \times 10^{-2}, F_{2}$ has been measured over a significant range in $Q^{2}$.

For most hard-scattering applications in particle physics, it is straightforward to begin with parametrizations of parton distribution functions tied to data at modest values of $Q^{2}$ and evolve them to the desired high scale using the Altarelli-Parisi equations [50]. The special challenge of UHE neutrino-nucleon scattering is that the $W$-boson propagator emphasizes smaller and smaller values of $x$ as the neutrino energy $E_{\nu}$ increases. In the UHE domain, the most important contributions to the $\nu N$ cross section come from $x \sim M_{W}^{2} / 2 M E_{\nu}$. Up to $E_{\nu} \approx 10^{5} \mathrm{GeV}$, the parton distributions are sampled only at values of $x$ where they have been constrained by experiment. At still higher energies, we require parton distributions at such small values of $x$ that direct experimental constraints are not available, not even at low values of $Q^{2}$.

The theoretical uncertainties that enter the evaluation of the UHE neutrinonucleon cross section arise from the low- $Q^{2}$ parametrization, the evolution of the parton distribution functions to large values of $Q^{2} \sim M_{W}^{2}$, and the extrapolation to small values of $x$. The greatest uncertainty is due to the small- $x$ extrapolation.

Because experiments are limited to values of $x \gtrsim 10^{-4}$, fits to structure functions have to be based on plausible but poorly constrained extrapolations to $x=0$. The parton distributions are traditionally obtained by assuming com- 
pact forms at $Q^{2}=Q_{0}^{2}=$ a few $\mathrm{GeV}^{2}$ :

$$
\begin{aligned}
& x q_{v}\left(x, Q_{0}^{2}\right)=A_{v} x^{\beta_{v}}(1-x)^{\eta_{v}} f_{v}(\sqrt{x}), \\
& x q_{s}\left(x, Q_{0}^{2}\right)=A_{s} x^{-\lambda}(1-x)^{\eta_{s}} f_{s}(\sqrt{x}), \\
& x G\left(x, Q_{0}^{2}\right)=A_{g} x^{-\lambda}(1-x)^{\eta_{g}} f_{g}(\sqrt{x}),
\end{aligned}
$$

where $q_{v}$ is a valence-quark distribution, $q_{s}$ is a sea-quark distribution, and $G(x)$ is the gluon distribution. The functions $f_{i}(\sqrt{x})$ are polynomials in $\sqrt{x}$ that satisfy $f_{i}(0)=1$. Sum rules provide broad constraints on the parameters. For example, the requirement that the momentum integral of the gluon distribution be finite means that $x G\left(x, Q^{2}\right)$ must be less singular than $x^{-1}$ at $x=0$. The parameters are determined from fits to experimental data and the resulting forms are evolved to higher values of $Q^{2}$ using the next-to-leading order Altarelli-Parisi equations. We employ in this work the latest (CTEQ3) of the parton distributions determined by the CTEQ collaboration [51] and several sets (MRS A', G, D_, and D_') from the family of parton distributions produced by Martin, Roberts, and Stirling [52-55]. Both the CTEQ and MRS parametrizations result from global fits to vast data sets and obey sum-rule constraints.

Currently there are two theoretical approaches, both based on perturbative QCD, to understanding the $Q^{2}$-evolution of small- $x$ parton distributions. The traditional approach, followed in the CTEQ3 [51] and in the MRS A' [52] and $\mathrm{G}$ [53] distributions, is to determine parton densities for $Q^{2}>Q_{0}^{2}$ by solving the next-to-leading-order Altarelli-Parisi evolution equations numerically. The second approach to small- $x$ evolution is to solve the Balitskil-Fadin-KuraevLipatov (BFKL) equation, which is effectively a leading $\alpha_{s} \ln (1 / x)$ resummation of soft gluon emissions [56]. In practical terms, the small- $x$ behavior is an input at some scale $Q_{0}^{2}$ in the traditional approach, and a dynamically generated output in the BFKL scheme. The BFKL approach predicts a singular behavior in $x$ and a rapid $Q^{2}$-variation,

$$
x q_{s}\left(x, Q^{2}\right) \sim \sqrt{Q^{2}} x^{-0.5}
$$

Applying the Altarelli-Parisi equations to singular input distributions $\propto x^{-\frac{1}{2}}$ leads to

$$
x q_{s}\left(x, Q^{2}\right) \sim \ln \left(Q^{2}\right) x^{-0.5}
$$

a less rapid growth with $Q^{2}$.

The Altarelli-Parisi approach is applicable in the not-so-small- $x$ and large$Q^{2}$ region, while the BFKL solution applies to the small- $x$ and moderate- $Q^{2}$ 
region. BFKL evolution eventually breaks down at large $Q^{2}$, because of the rapid growth exhibited in (4). In the case of ultrahigh-energy neutrino-nucleon interactions, the region of interest is small- $x$ and large- $Q^{2}$, which requires a resummation of both $\ln 1 / x$ and $\ln Q^{2} / Q_{0}^{2}$ contributions. Although some progress has been made in developing a "unified" evolution equation $[57,58]$, the full solution and global fits to data are far from being achieved.

The standard Altarelli-Parisi evolution of the parton distribution functions is applicable for the calculation of the total neutrino cross section up to $E_{\nu} \approx 10^{5}$ $\mathrm{GeV}$, so it is a reasonable starting point for calculating the cross section for higher energy neutrinos. Consequently, the calculation of the total neutrinonucleon cross section presented here relies on the CTEQ3 and MRS A' parton distributions obtained using next-to-leading-order (NLO) evolution equations. The CTEQ3 distribution functions, depending on the order of the evolution and the factorization scheme, use $\lambda \simeq 0.28-0.35$, while $\lambda=0.17$ for MRS A'. The CTEQ3 distributions are particularly convenient as a benchmark because the numerical evolution is provided for $x \rightarrow 0$, including the region in which the Altarelli-Parisi equations may not be reliable. (The MRS A' distributions are available for $x \geq 10^{-5}$.) We use the CTEQ3 parton distributions, with NLO evolution from $Q_{0}=1.6 \mathrm{GeV}$, as our canonical set. We choose the deepinelastic scattering factorization scheme (DIS) parametrization of the parton distribution functions, for which the exponent $\lambda=0.332$. Results calculated with this set of parton distributions are labeled as CTEQ-DIS in the discussion below.

To estimate the uncertainty in the small- $x$ parton distributions evaluated at $Q^{2} \sim M_{W}^{2}$, we consider alternative treatments of the small- $x$ behavior. To explore a less singular alternative, we extrapolate to $x=0$ using the double-logarithmic-approximation (DLA) [59], an approximate solution to the Altarelli-Parisi equations for not-too-singular input distributions. The form of the sea-quark distribution is $[59,60]$

$$
x q_{s}\left(x, Q^{2}\right)=C\left(Q^{2}\right) \sqrt{\frac{2\left(\xi-\xi_{0}\right)}{\rho}} \exp \left\{\left[\left(2 \rho\left(\xi-\xi_{0}\right)\right]^{1 / 2}\right\} .\right.
$$

Here, $\rho=\left(8 N / b_{0}\right) \ln (1 / x), \xi(Q)=\ln \ln \left(Q^{2} / \Lambda^{2}\right), N=3$ is the number of colors, and $b_{0}=\left(11 N-2 n_{f}\right) / 3$ for $n_{f}$ flavors. This form was used in Ref. [37,38] to extrapolate the EHLQ parton distributions [41] below $x^{\mathrm{min}}=10^{-4}$. The EHLQ distributions $x q_{s}\left(x, Q_{0}^{2}\right)$ are finite as $x \rightarrow 0$, i.e., correspond to $\lambda=0$ in (4) [61]. To estimate a lower limit on the UHE $\nu N$ cross section, we use the DLA form of Eq. (6) for $x<x^{\min }=10^{-4}$ as an extrapolation of the leadingorder parametrization of the CTEQ3 parton distribution functions (labeled CTEQ-DLA). Here, following the procedure of Ref. [37], we choose $C\left(Q^{2}\right)$ to match $x^{\mathrm{min}} q_{s}\left(x^{\mathrm{min}}, Q^{2}\right)$ from CTEQ-LO. The five-flavor value of $\Lambda_{\mathrm{QCD}}$ is $\Lambda_{\mathrm{QCD}}^{L O}=132 \mathrm{MeV}[62]$. 


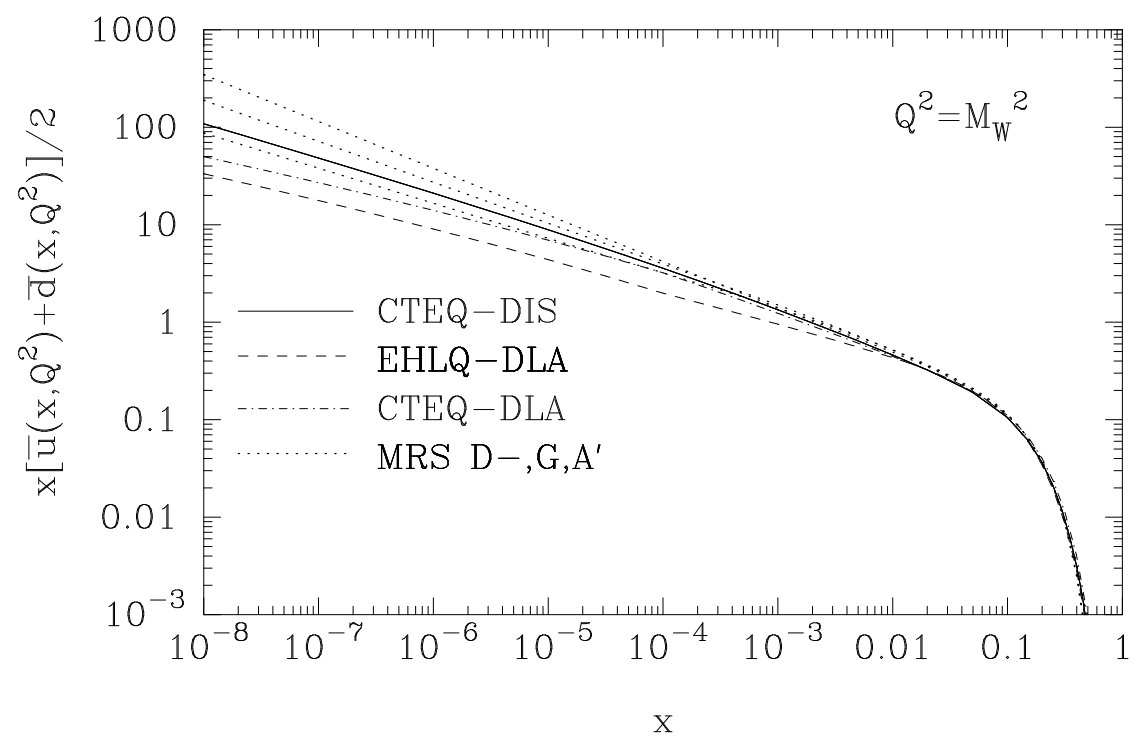

Fig. 1. Comparison of the light-quark sea at $Q^{2}=M_{W}^{2}$ for various parton distributions. Of the MRS distributions, D_ (A') is the most (least) singular.

A more singular form of the parton distributions at small- $x$, motivated by BFKL dynamics, appears in the MRS D_ set [54]. In the limit of very small $x$, the behavior of the MRS D_ sea is $x q_{s}\left(x, Q_{0}^{2}\right)=C\left(Q_{0}^{2}\right) x^{-0.5}$. These distributions appear to slightly overestimate the low- $Q^{2}$ HERA data $[48,49]$ in the interval $10^{-4} \lesssim x \lesssim 10^{-2}$. Thus, for large $E_{\nu}$, they can provide a reasonable upper limit on the cross section [63].

To illustrate the range of parton distributions that these choices represent, we plot the light-quark sea distribution $x(\bar{u}+\bar{d}) / 2$ versus $x$ for $Q^{2}=M_{W}^{2}$ in Figure 1. For $x \gtrsim 10^{-4}$, the MRS and CTEQ distributions all are in close agreement. This consonance allows us to make confident predictions of the $\nu N$ cross sections for neutrino energies up to about $10^{6} \mathrm{GeV}$. The spread in the parton distributions at smaller values of $x$ reflects the uncertain extrapolation toward $x=0$.

\section{Neutrino-Nucleon Interactions}

It is straightforward to calculate the inclusive cross section for the reaction

$$
\nu_{\mu} N \rightarrow \mu^{-}+\text {anything }
$$

where $N \equiv \frac{n+p}{2}$ is an isoscalar nucleon, in the renormalization groupimproved parton model. The differential cross section is written in terms of 
the Bjorken scaling variables $x=Q^{2} / 2 M \nu$ and $y=\nu / E_{\nu}$ as

$$
\frac{d^{2} \sigma}{d x d y}=\frac{2 G_{F}^{2} M E_{\nu}}{\pi}\left(\frac{M_{W}^{2}}{Q^{2}+M_{W}^{2}}\right)^{2}\left[x q\left(x, Q^{2}\right)+x \bar{q}\left(x, Q^{2}\right)(1-y)^{2}\right]
$$

where $-Q^{2}$ is the invariant momentum transfer between the incident neutrino and outgoing muon, $\nu=E_{\nu}-E_{\mu}$ is the energy loss in the lab (target) frame, $M$ and $M_{W}$ are the nucleon and intermediate-boson masses, and $G_{F}=1.16632 \times$ $10^{-5} \mathrm{GeV}^{-2}$ is the Fermi constant. The quark distribution functions are

$$
\begin{aligned}
q\left(x, Q^{2}\right)= & \frac{u_{v}\left(x, Q^{2}\right)+d_{v}\left(x, Q^{2}\right)}{2}+\frac{u_{s}\left(x, Q^{2}\right)+d_{s}\left(x, Q^{2}\right)}{2} \\
& +s_{s}\left(x, Q^{2}\right)+b_{s}\left(x, Q^{2}\right) \\
\bar{q}\left(x, Q^{2}\right)= & \frac{u_{s}\left(x, Q^{2}\right)+d_{s}\left(x, Q^{2}\right)}{2}+c_{s}\left(x, Q^{2}\right)+t_{s}\left(x, Q^{2}\right),
\end{aligned}
$$

where the subscripts $v$ and $s$ label valence and sea contributions, and $u, d, c$, $s, t, b$ denote the distributions for various quark flavors in a proton. At the energies of interest for neutrino astronomy, perturbative QCD corrections to the cross section formula (8) are insignificant, so we omit them. In particular, in the DIS factorization scheme (the CTEQ-DIS parton distributions), the terms proportional to $\alpha_{s}$ [65] in the NLO cross section contribute only a few percent.

Because of the great mass of the top quark, $t \bar{t}$ pairs are a negligible component of the nucleon over the $Q^{2}$-range relevant to neutrino-nucleon scattering. Consequently we drop the contribution of the top sea. At the energies of interest here, it is a sound kinematical simplification to treat charm and bottom quarks as massless. However, the threshold suppression of the $b \rightarrow t$ transition must be taken into account. We adopt the standard "slow-rescaling" prescription [64], with $m_{t}=175 \mathrm{GeV} / c^{2}$. Numerical integrations were carried out using the adaptive Monte Carlo routine VEGAS [66], and Gaussian techniques.

We show in Figure 2 the contributions of valence quarks and of the different quark flavors in the sea to the $\nu N$ charged-current total cross section, according to the CTEQ3 parton distributions. As expected, the valence contribution dominates at low energies. There, in the parton-model idealization that quark distributions are independent of $Q^{2}$, differential and total cross sections are proportional to the neutrino energy. Up to energies $E_{\nu} \sim 10^{11} \mathrm{eV}$, the familiar manifestation of the QCD evolution of the parton distributions is to degrade the valence component, and so to decrease the total cross section. At still higher energies, the gauge-boson propagator restricts $Q^{2}=2 M E_{\nu} x y$ to values near $M_{W}^{2}$, and so limits the effective interval in $x$ to the region around 


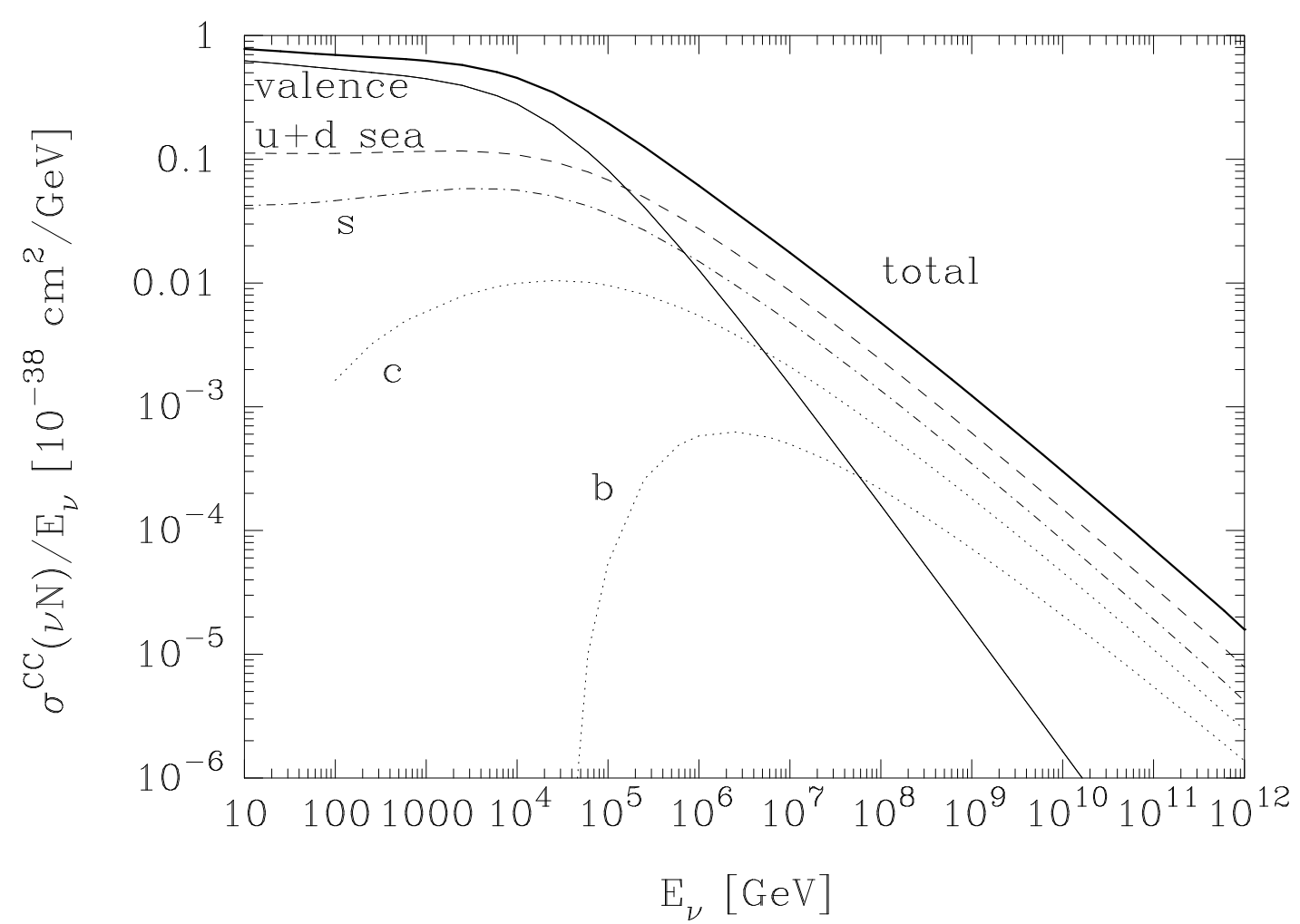

Fig. 2. Components of the $\nu N$ charged-current cross section as functions of the neutrino energy for the CTEQ3 distributions.

$M_{W}^{2} / 2 M E_{\nu}\langle y\rangle$. Figure 3 shows the contributions to the cross section from different regions of $x$. At modest values of $Q^{2}$, the effect of this $W$-propagator damping is to further diminish the cross section below the point-coupling, parton-model approximation. Above about $10^{16} \mathrm{eV}$, the valence contribution is even smaller than the contribution of the $b \bar{b}$ sea.

A second effect of QCD evolution is to increase the population of heavy quarks $(s, c, b)$ within the proton, and to increase the importance of the light-quark sea at small values of $x$. Andreev, Berezinsky, and Smirnov [67] have pointed out that the effect of this growth in the density of the parton sea is to enhance the cross section at high energies. This effect is apparent in Figure 4. There we compare the CTEQ3 cross section with the 1986 cross section [37] based on the EHLQ structure functions and with the case of no evolution. We see that the EHLQ-based cross section is enhanced by fully an order of magnitude at high energies by the evolution of the sea. At low energies, the decrease in the cross section brought about by the degradation of the valence distribution is apparent in the comparison of the EHLQ curves with and without evolution. We also show in Figure 4 the CTEQ3 prediction for the $\bar{\nu} N$ charged-current cross section. At the highest energies, where the contributions of valence quarks are unimportant, the neutrino and antineutrino cross sections are identical. 
Our new evaluation of the $\nu N$ cross section differs from the earlier calculations at both low and high energies. At both extremes, the difference is owed to changes in our understanding of parton distribution functions. The EHLQ

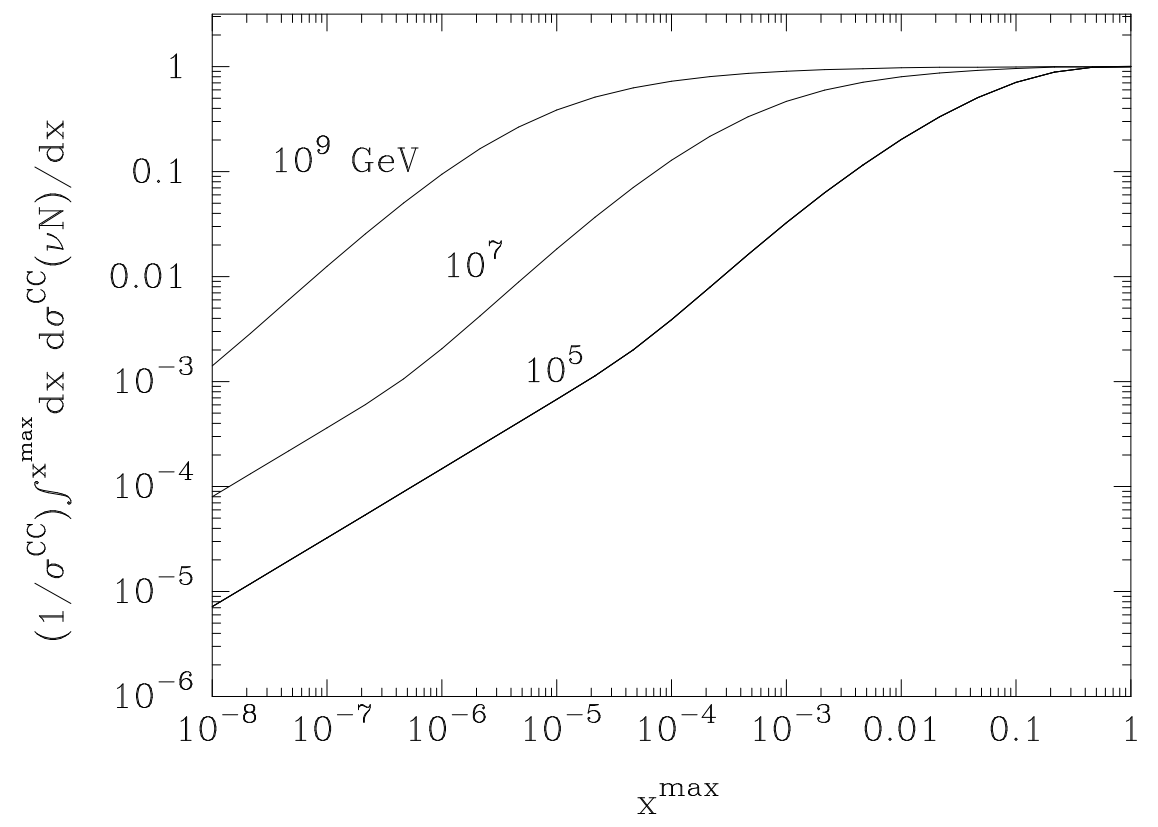

Fig. 3. Integral cross section $(1 / \sigma) \int_{0}^{x^{\max }} d x d \sigma / d x$ for the charged-current reaction $\nu_{\mu} N \rightarrow \mu^{-}+$anything at $E_{\nu}=10^{5}, 10^{7}$, and $10^{9} \mathrm{GeV}$. As the neutrino energy increases, the dominant contributions come from smaller values of $x$.

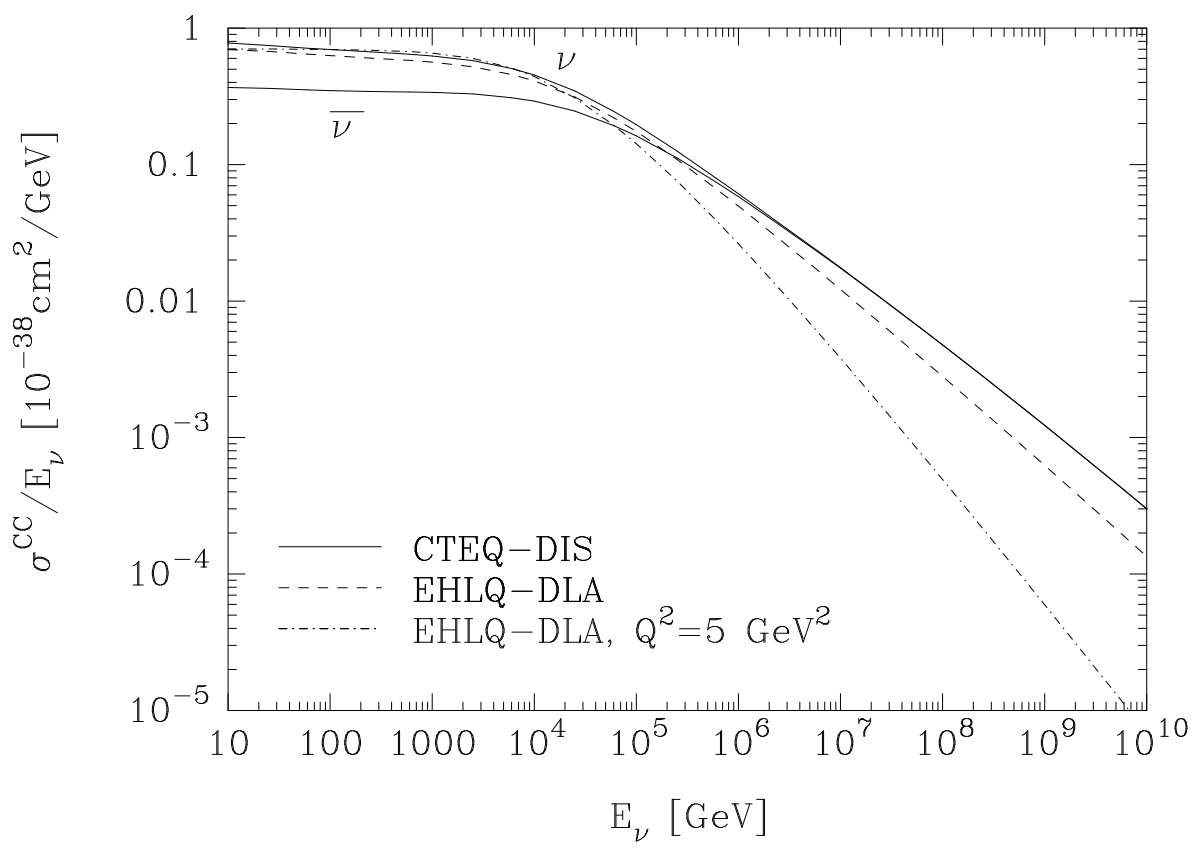

Fig. 4. Energy dependence of the $\nu N$ and $\bar{\nu} N$ charged-current cross sections according to the CTEQ3 parton distributions. The EHLQ-DLA prediction [37] for the $\nu N$ cross section is also shown, together with the $\nu N$ cross section based on the unevolved EHLQ structure functions with $Q^{2}$ fixed at $Q_{0}^{2}=5 \mathrm{GeV}^{2}$. 
parton distributions, on which the earlier calculations were based, were based on the CERN-Dortmund-Heidelberg-Saclay measurements of neutrino-nucleon structure functions [68]. We now know that the normalization of the CDHS structure functions was about $15 \%$ low [69]. The change in normalization directly affects the cross sections at low energies. At higher energies, which are sensitive to small values of $x$, the shape of the parton distribution as $x \rightarrow 0$ is decisive. At low $Q^{2}$, the EHLQ distributions $x q_{s}(x)$ are finite as $x \rightarrow 0$, whereas HERA experiments point to singular behavior, parametrized in the CTEQ distributions as $x q_{s}(x) \rightarrow x^{-0.332}$. The density of partons at small values of $x$ and modest values of $Q^{2}$ is thus greater than was assumed in the earlier work.

We show in Figure 5 the charged-current $\nu N$ cross section implied by several sets of parton distributions derived from global fits. There is excellent agreement among the predictions of the MRS D_, G, and A' distributions and the CTEQ3 distributions up to $E_{\nu} \approx 10^{7} \mathrm{GeV}$. Above that energy, our DLA modification of the CTEQ3 distributions gives a lower cross section than the full CTEQ3 distributions (CTEQ-DIS), as expected from its less singular behavior as $x \rightarrow 0$. At the highest energy displayed, the most singular (MRS D_) distribution predicts a significantly higher cross section than the others. Above about $10^{6} \mathrm{GeV}$, the EHLQ-DLA distributions yield noticeably smaller cross sections than the modern distributions. All the MRS and CTEQ curves are in reasonable agreement with the HERA measurement [70] of the chargedcurrent cross section at an equivalent neutrino energy of $46.7 \mathrm{TeV}$ [71]. The parton distributions inferred by Frichter, et al. from HERA data [44] yield cross sections that stand apart from those derived from global fits.

Two other groups recently have evaluated the neutrino-nucleon charged-current cross sections at high energies. Parente and Zas [72] used the MRS G distributions [53] to compute $\sigma_{\mathrm{CC}}(\nu N)$ for neutrino energies in the range $200 \mathrm{GeV} \leq$ $E_{\nu} \leq 10^{7} \mathrm{GeV}$, in which no special treatment of the $x \rightarrow 0$ behavior of the parton distributions is required. The results presented in their Figure 2 agree with the corresponding curve in Figure 5 above. Butkevich, et al. [73] have evaluated $\sigma_{\mathrm{CC}}(\nu N)$ and $\sigma_{\mathrm{CC}}(\bar{\nu} N)$ for $10^{2} \mathrm{GeV} \leq E_{\nu} \leq 10^{6} \mathrm{GeV}$ using the MRS A distributions [52], an early version of the Glück-Reya-Vogt distributions [74], and the Morfin-Tung ancestor [75] of the CTEQ3 distributions we use. The results presented in their Figure 1 agree with those in our Figure 5. Butkevich, et al. have also explored two extrapolations of the MRS A parton distributions to very small values of $x$. The values of $\sigma_{\mathrm{CC}}(\nu N)$ presented in their Table 1 for $10^{7} \mathrm{GeV} \leq E_{\nu} \leq 10^{12} \mathrm{GeV}$ are close to those we give in Table 1 below for our nominal set, the CTEQ3 distributions, and agree well with our calculations using the MRS A' distributions.

The differential cross section $\left(1 / E_{\nu}\right) d \sigma / d y$ for neutrino-nucleon scattering is shown in Figure 6 . The peaking of the cross section near $y=0$, which becomes 
increasingly prominent with increasing neutrino energy, is a direct consequence of the cutoff in $Q^{2}$ enforced by the $W$ propagator. However, because of the growth of the quark distributions as small values of $x$ for large $Q^{2}$, the cross section is nonnegligible at finite values of $y$. Accordingly, the mean inelasticity $\langle y\rangle$ does not decrease rapidly as the energy increases. This parameter is shown for both neutrinos and antineutrinos in Figure 7.

A parallel calculation leads to the neutral-current cross section. In this case the differential cross section for the reaction $\nu_{\mu} N \rightarrow \nu+$ anything is given by

$$
\frac{d^{2} \sigma}{d x d y}=\frac{G_{F}^{2} M E_{\nu}}{2 \pi}\left(\frac{M_{Z}^{2}}{Q^{2}+M_{Z}^{2}}\right)^{2}\left[x q^{0}\left(x, Q^{2}\right)+x \bar{q}^{0}\left(x, Q^{2}\right)(1-y)^{2}\right]
$$

where $M_{Z}$ is the mass of the neutral intermediate boson. The quantities involving parton distribution functions are

$$
\begin{aligned}
& q^{0}\left(x, Q^{2}\right)=\left[\frac{u_{v}\left(x, Q^{2}\right)+d_{v}\left(x, Q^{2}\right)}{2}+\frac{u_{s}\left(x, Q^{2}\right)+d_{s}\left(x, Q^{2}\right)}{2}\right]\left(L_{u}^{2}+L_{d}^{2}\right) \\
& \quad+\left[\frac{u_{s}\left(x, Q^{2}\right)+d_{s}\left(x, Q^{2}\right)}{2}\right]\left(R_{u}^{2}+R_{d}^{2}\right)+ \\
& {\left[s_{s}\left(x, Q^{2}\right)+b_{s}\left(x, Q^{2}\right)\right]\left(L_{d}^{2}+R_{d}^{2}\right)+\left[c_{s}\left(x, Q^{2}\right)+t_{s}\left(x, Q^{2}\right)\right]\left(L_{u}^{2}+R_{u}^{2}\right)}
\end{aligned}
$$

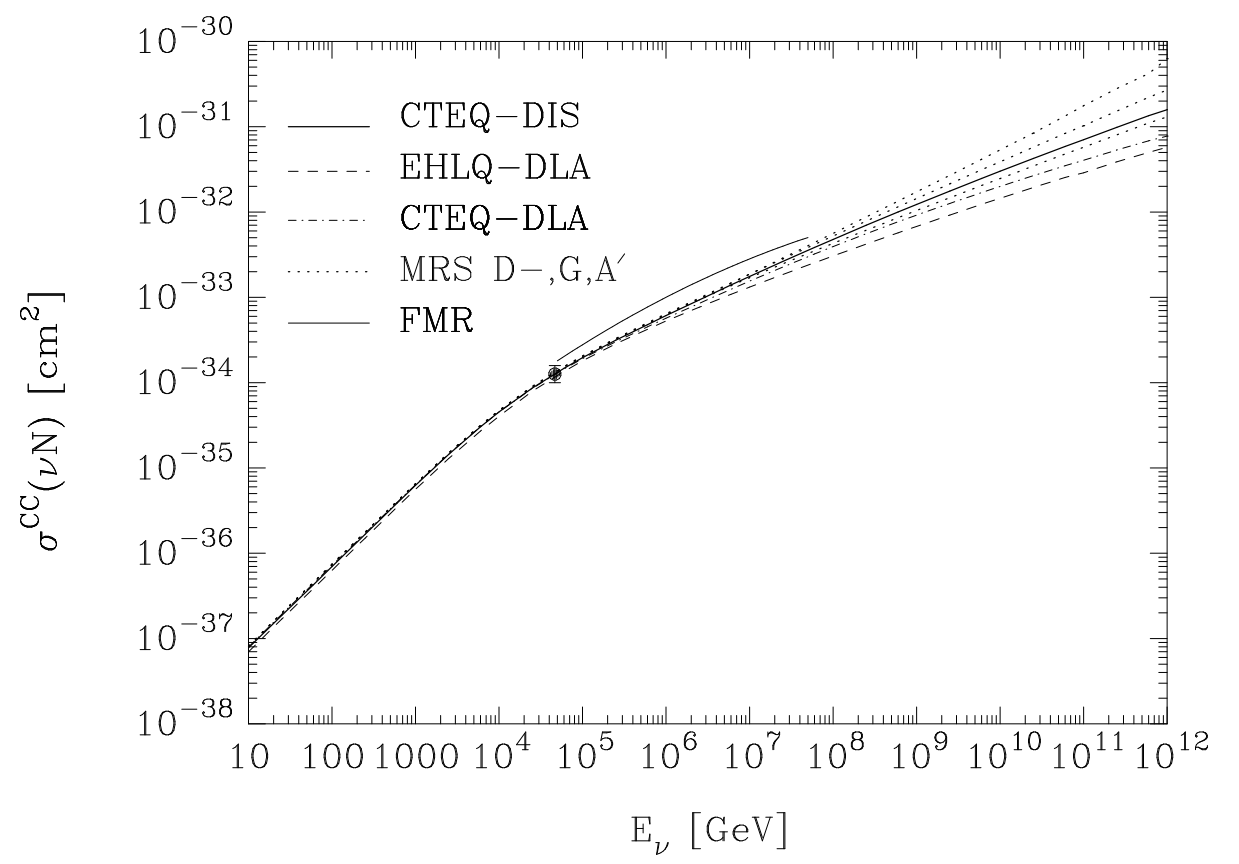

Fig. 5. The charged-current cross section for $\nu_{\mu}$ interactions with an isoscalar nucleon. The parametrization of Frichter, et al. [44] is shown for $5 \times 10^{4} \mathrm{GeV}<E_{\nu}<5 \times 10^{7} \mathrm{GeV}$. The data point is an average of measurements by the ZEUS and H1 Collaborations at HERA [70]. 


$$
\begin{gathered}
\bar{q}^{0}\left(x, Q^{2}\right)=\left[\frac{u_{v}\left(x, Q^{2}\right)+d_{v}\left(x, Q^{2}\right)}{2}+\frac{u_{s}\left(x, Q^{2}\right)+d_{s}\left(x, Q^{2}\right)}{2}\right]\left(R_{u}^{2}+R_{d}^{2}\right) \\
+\left[\frac{u_{s}\left(x, Q^{2}\right)+d_{s}\left(x, Q^{2}\right)}{2}\right]\left(L_{u}^{2}+L_{d}^{2}\right)+ \\
{\left[s_{s}\left(x, Q^{2}\right)+b_{s}\left(x, Q^{2}\right)\right]\left(L_{d}^{2}+R_{d}^{2}\right)+\left[c_{s}\left(x, Q^{2}\right)+t_{s}\left(x, Q^{2}\right)\right]\left(L_{u}^{2}+R_{u}^{2}\right),}
\end{gathered}
$$

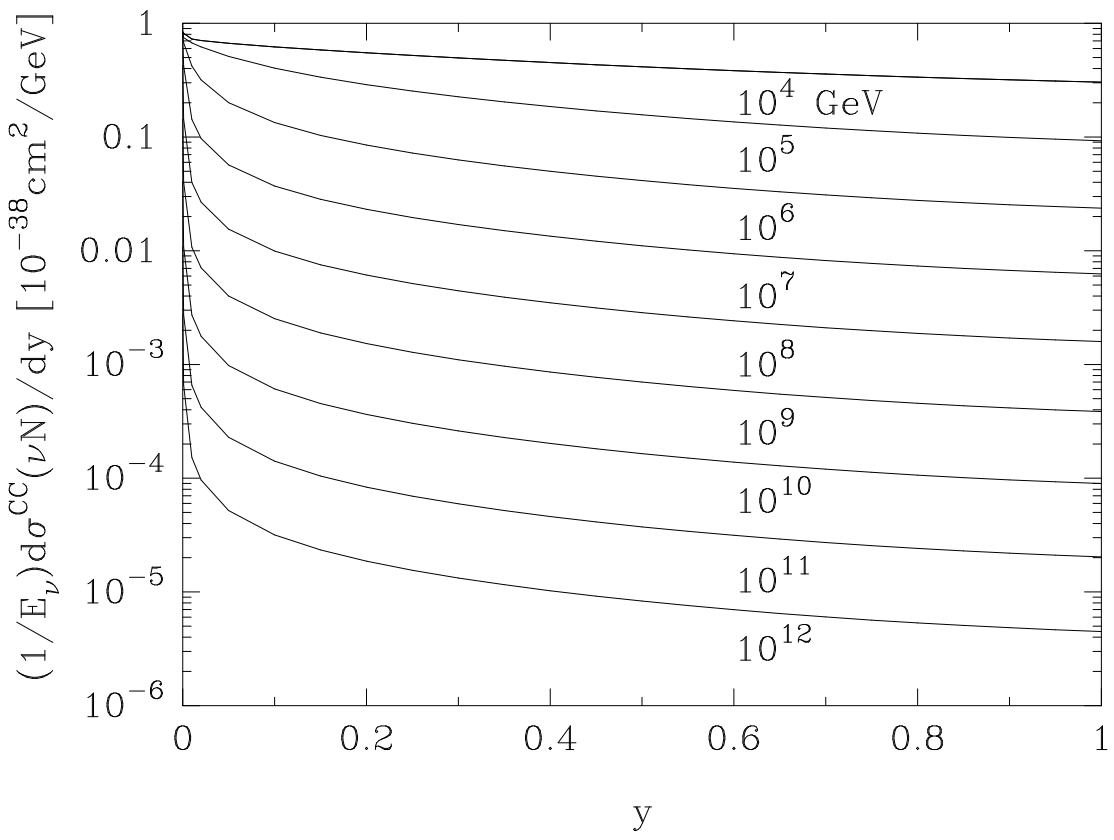

Fig. 6. Differential cross section for $\nu N$ scattering for neutrino energies between $10^{4} \mathrm{GeV}$ and $10^{12} \mathrm{GeV}$.

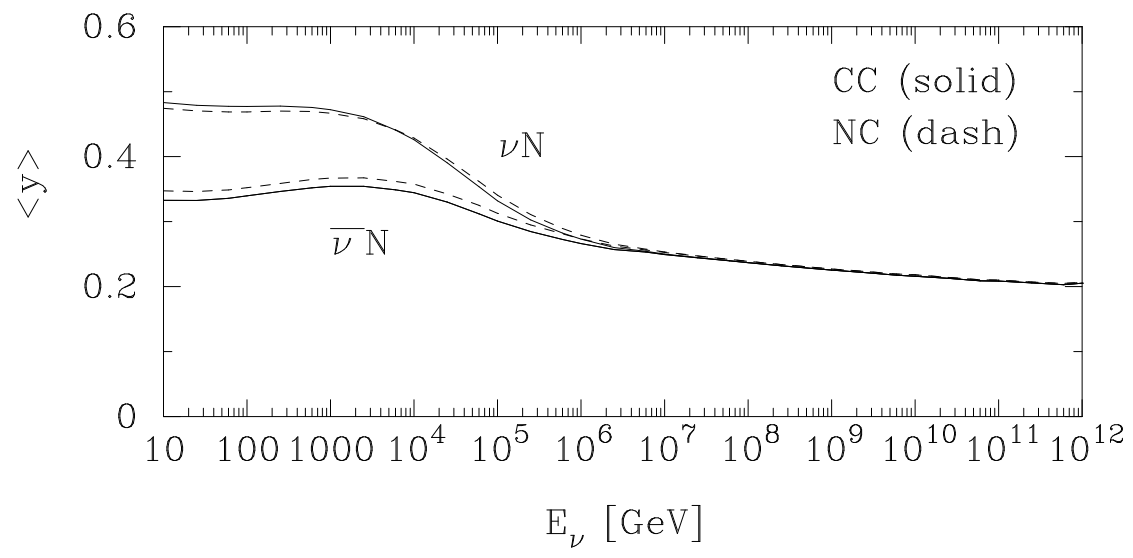

Fig. 7. Energy dependence of the inelasticity parameter $y$ for charged-current (solid lines) and neutral-current (dashed lines) interactions as a function of the incident neutrino energy. 
where the chiral couplings are

$$
\begin{array}{ll}
L_{u}=1-\frac{4}{3} x_{W} & L_{d}=-1+\frac{2}{3} x_{W} \\
R_{u}=-\frac{4}{3} x_{W} & R_{d}=\frac{2}{3} x_{W}
\end{array}
$$

and $x_{W}=\sin ^{2} \theta_{W}$ is the weak mixing parameter. For numerical calculations we have chosen $x_{W}=0.226$ [76]. Again the top-quark sea is negligible.

Cross sections for neutral-current scattering of neutrinos and antineutrinos from isoscalar nucleons are shown as the dashed curves in Figures 8 and 9, respectively. There we also show the charged-current cross sections (as thin solid curves) and the sum of charged-current and neutral-current cross sections (as thick solid curves).

Numerical values of the cross sections and inelasticity parameters, which characterize the angular distribution of outgoing leptons, are indispensable for simulating the degradation of the neutrino flux passing through the Earth, and for calculating event rates in proposed detectors. We have gathered in Tables 1 and 2 the charged-current and neutral-current cross sections and values of $\langle y\rangle$, for $\nu N$ and $\bar{\nu} N$ collisions, respectively.

For neutrino energies in the range $10^{15} \mathrm{eV} \leq E_{\nu} \leq 10^{21} \mathrm{eV}$, good representations of the cross sections are given by simple power-law forms:

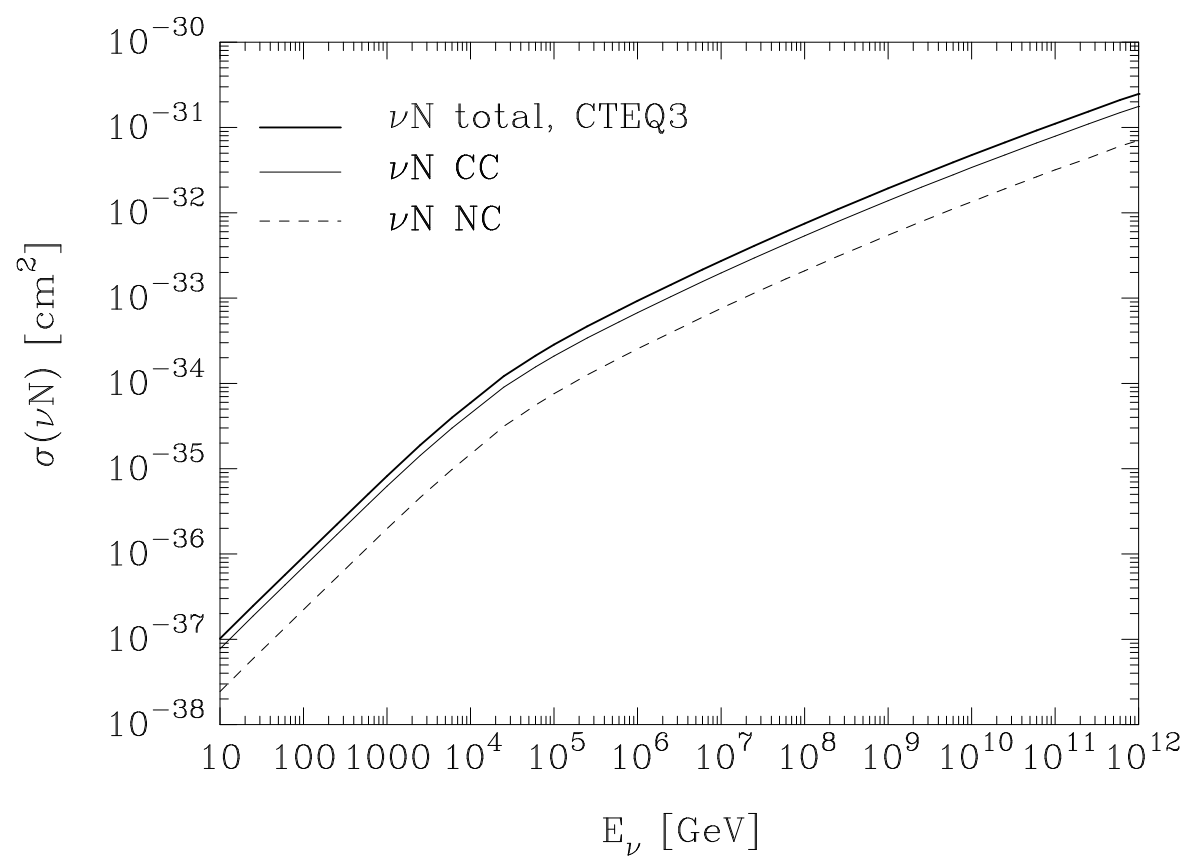

Fig. 8. Cross sections for $\nu N$ interactions at high energies: dotted line, $\sigma(\nu N \rightarrow \nu+$ anything $)$; thin line, $\sigma\left(\nu N \rightarrow \mu^{-}+\right.$anything $) ;$thick line, total (charged-current plus neutral-current) cross section. 
Table 1

Charged-current and neutral-current cross sections for $\nu N$ interactions, and the corresponding values of the mean inelasticity $\langle y\rangle$, for the CTEQ-DIS distributions.

\begin{tabular}{ccccc}
\hline$E_{\nu}[\mathrm{GeV}]$ & $\sigma_{\mathrm{CC}}\left[\mathrm{cm}^{2}\right]$ & $\sigma_{\mathrm{NC}}\left[\mathrm{cm}^{2}\right]$ & $\langle y\rangle_{\mathrm{CC}}$ & $\langle y\rangle_{\mathrm{NC}}$ \\
\hline $10^{1}$ & $0.777 \times 10^{-37}$ & $0.242 \times 10^{-37}$ & 0.483 & 0.474 \\
$10^{2}$ & $0.697 \times 10^{-36}$ & $0.217 \times 10^{-36}$ & 0.477 & 0.470 \\
$10^{3}$ & $0.625 \times 10^{-35}$ & $0.199 \times 10^{-35}$ & 0.472 & 0.467 \\
$10^{4}$ & $0.454 \times 10^{-34}$ & $0.155 \times 10^{-34}$ & 0.426 & 0.428 \\
$10^{5}$ & $0.196 \times 10^{-33}$ & $0.745 \times 10^{-34}$ & 0.332 & 0.341 \\
$10^{6}$ & $0.611 \times 10^{-33}$ & $0.252 \times 10^{-33}$ & 0.273 & 0.279 \\
$10^{7}$ & $0.176 \times 10^{-32}$ & $0.748 \times 10^{-33}$ & 0.250 & 0.254 \\
$10^{8}$ & $0.478 \times 10^{-32}$ & $0.207 \times 10^{-32}$ & 0.237 & 0.239 \\
$10^{9}$ & $0.123 \times 10^{-31}$ & $0.540 \times 10^{-32}$ & 0.225 & 0.227 \\
$10^{10}$ & $0.301 \times 10^{-31}$ & $0.134 \times 10^{-31}$ & 0.216 & 0.217 \\
$10^{11}$ & $0.706 \times 10^{-31}$ & $0.316 \times 10^{-31}$ & 0.208 & 0.210 \\
$10^{12}$ & $0.159 \times 10^{-30}$ & $0.715 \times 10^{-31}$ & 0.205 & 0.207 \\
\hline
\end{tabular}

Table 2

Charged-current and neutral-current cross sections for $\bar{\nu} N$ interactions, and the corresponding values of the mean inelasticity $\langle y\rangle$, for the CTEQ-DIS distributions.

\begin{tabular}{ccccc}
\hline$E_{\nu}[\mathrm{GeV}]$ & $\sigma_{\mathrm{CC}}\left[\mathrm{cm}^{2}\right]$ & $\sigma_{\mathrm{NC}}\left[\mathrm{cm}^{2}\right]$ & $\langle y\rangle_{\mathrm{CC}}$ & $\langle y\rangle_{\mathrm{NC}}$ \\
\hline $10^{1}$ & $0.368 \times 10^{-37}$ & $0.130 \times 10^{-37}$ & 0.333 & 0.350 \\
$10^{2}$ & $0.349 \times 10^{-36}$ & $0.122 \times 10^{-36}$ & 0.340 & 0.354 \\
$10^{3}$ & $0.338 \times 10^{-35}$ & $0.120 \times 10^{-35}$ & 0.354 & 0.368 \\
$10^{4}$ & $0.292 \times 10^{-34}$ & $0.106 \times 10^{-34}$ & 0.345 & 0.358 \\
$10^{5}$ & $0.162 \times 10^{-33}$ & $0.631 \times 10^{-34}$ & 0.301 & 0.313 \\
$10^{6}$ & $0.582 \times 10^{-33}$ & $0.241 \times 10^{-33}$ & 0.266 & 0.273 \\
$10^{7}$ & $0.174 \times 10^{-32}$ & $0.742 \times 10^{-33}$ & 0.249 & 0.253 \\
$10^{8}$ & $0.477 \times 10^{-32}$ & $0.207 \times 10^{-32}$ & 0.237 & 0.239 \\
$10^{9}$ & $0.123 \times 10^{-31}$ & $0.540 \times 10^{-32}$ & 0.225 & 0.227 \\
$10^{10}$ & $0.301 \times 10^{-31}$ & $0.134 \times 10^{-31}$ & 0.216 & 0.217 \\
$10^{11}$ & $0.706 \times 10^{-31}$ & $0.316 \times 10^{-31}$ & 0.208 & 0.210 \\
$10^{12}$ & $0.159 \times 10^{-30}$ & $0.715 \times 10^{-31}$ & 0.205 & 0.207 \\
\hline
\end{tabular}




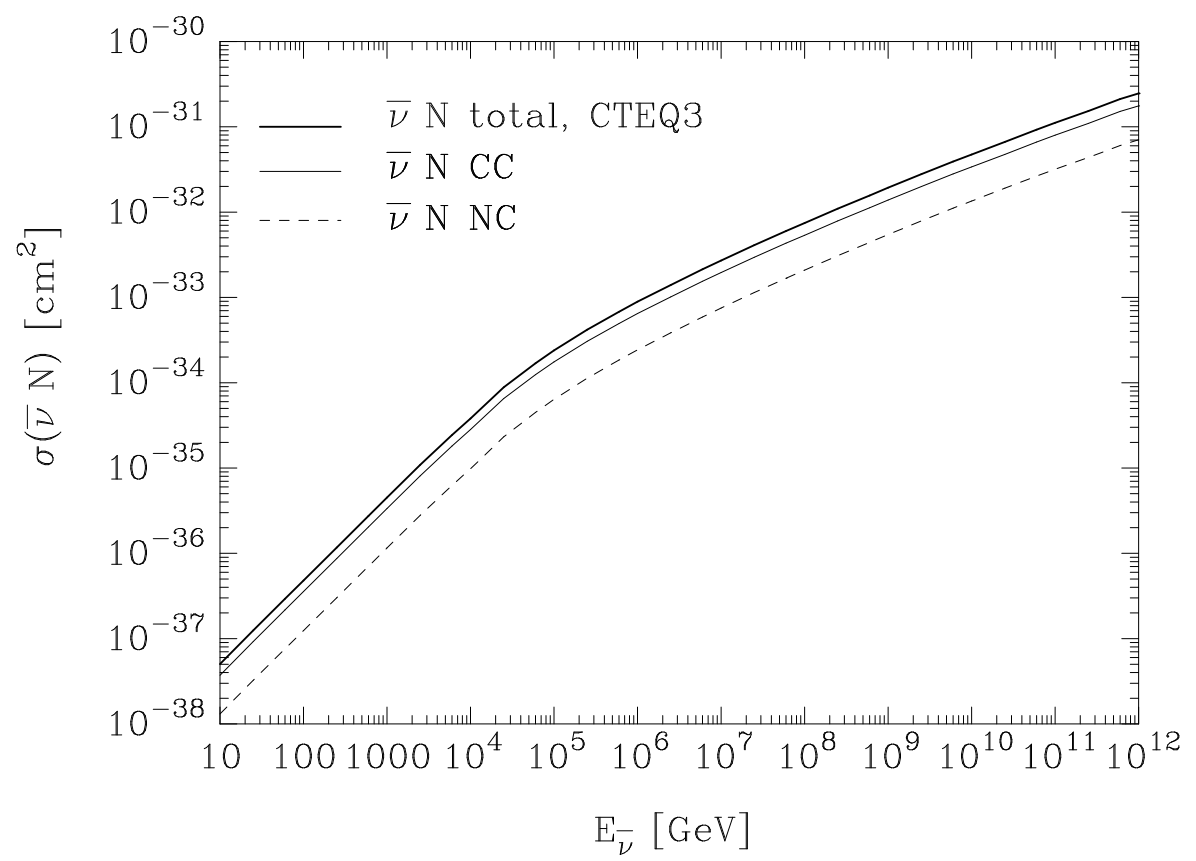

Fig. 9. Cross sections for $\bar{\nu} N$ interactions at high energies: dotted line, $\sigma(\bar{\nu} N \rightarrow \bar{\nu}+$ anything $) ;$ thin line, $\sigma\left(\bar{\nu} N \rightarrow \mu^{+}+\right.$anything $) ;$thick line, total (charged-current plus neutral-current) cross section.

$$
\begin{aligned}
& \sigma_{\mathrm{CC}}(\nu N)=2.69 \times 10^{-36} \mathrm{~cm}^{2}\left(\frac{E_{\nu}}{1 \mathrm{GeV}}\right)^{0.402} \\
& \sigma_{\mathrm{NC}}(\nu N)=1.06 \times 10^{-36} \mathrm{~cm}^{2}\left(\frac{E_{\nu}}{1 \mathrm{GeV}}\right)^{0.408} \\
& \sigma_{\mathrm{CC}}(\bar{\nu} N)=2.53 \times 10^{-36} \mathrm{~cm}^{2}\left(\frac{E_{\nu}}{1 \mathrm{GeV}}\right)^{0.404} \\
& \sigma_{\mathrm{NC}}(\bar{\nu} N)=0.98 \times 10^{-36} \mathrm{~cm}^{2}\left(\frac{E_{\nu}}{1 \mathrm{GeV}}\right)^{0.410} .
\end{aligned}
$$

Before leaving the subject of neutrino-nucleon collisions, let us note that the cross sections for the reactions $\nu N \rightarrow W$ or $Z+$ anything are small compared with the cross sections for deeply inelastic scattering [77].

\section{Interaction of UHE Neutrinos with Electrons}

Because of the electron's small mass, neutrino-electron interactions can generally be neglected with respect to neutrino-nucleon interactions [78]. There is one exceptional case: resonant formation of the intermediate boson $W^{-}$in $\bar{\nu}_{e} e$ interactions at $6.3 \mathrm{PeV}[79]$. The resonant cross section is larger than the $\nu N$ cross section at any energy up to $10^{21} \mathrm{eV}$. Accordingly, it is important to have the neutrino-electron cross sections in mind when assessing the capabilities of 
neutrino telescopes.

Defining as usual the laboratory energy of the incoming neutrino as $E_{\nu}$ and the laboratory energy of the recoiling charged lepton as $E^{\prime}=y E_{\nu}$, we may write the differential cross sections for neutrino-electron scattering as [80]

$$
\begin{aligned}
& \frac{d \sigma\left(\nu_{\mu} e \rightarrow \nu_{\mu} e\right)}{d y}=\frac{G_{F}^{2} m E_{\nu}}{2 \pi} \frac{1}{\left(1+2 m E_{\nu} y / M_{Z}^{2}\right)^{2}}\left[R_{e}^{2}(1-y)^{2}+L_{e}^{2}\right] \\
& \frac{d \sigma\left(\bar{\nu}_{\mu} e \rightarrow \bar{\nu}_{\mu} e\right)}{d y}=\frac{G_{F}^{2} m E_{\nu}}{2 \pi} \frac{1}{\left(1+2 m E_{\nu} y / M_{Z}^{2}\right)^{2}}\left[R_{e}^{2}+L_{e}^{2}(1-y)^{2}\right] \\
& \frac{d \sigma\left(\nu_{\mu} e \rightarrow \mu \nu_{e}\right)}{d y}=\frac{G_{F}^{2} m E_{\nu}}{2 \pi} \frac{4\left[1-\left(\mu^{2}-m^{2}\right) / 2 m E_{\nu}\right]^{2}}{\left(1+2 m E_{\nu}(1-y) / M_{W}^{2}\right)^{2}} \\
& \frac{d \sigma\left(\nu_{e} e \rightarrow \nu_{e} e\right)}{d y}=\frac{G_{F}^{2} m E_{\nu}}{2 \pi}\left[\frac{R_{e}^{2}(1-y)^{2}}{\left(1+2 m E_{\nu} y / M_{Z}^{2}\right)^{2}}+\right. \\
& \left.\left(\frac{L_{e}}{1+2 m E_{\nu} y / M_{Z}^{2}}+\frac{2}{1+2 m E_{\nu}(1-y) / M_{W}^{2}}\right)^{2}\right] \text {, } \\
& \frac{d \sigma\left(\bar{\nu}_{e} e \rightarrow \bar{\nu}_{e} e\right)}{d y}=\frac{G_{F}^{2} m E_{\nu}}{2 \pi}\left[\frac{R_{e}^{2}}{\left(1+2 m E_{\nu} y / M_{Z}^{2}\right)^{2}}+\right. \\
& \left.\left|\frac{L_{e}}{1+2 m E_{\nu} y / M_{Z}^{2}}+\frac{2}{1-2 m E_{\nu} / M_{W}^{2}+i \Gamma_{W} / M_{W}}\right|^{2}(1-y)^{2}\right] \text {, } \\
& \frac{d \sigma\left(\bar{\nu}_{e} e \rightarrow \bar{\nu}_{\mu} \mu\right)}{d y}=\frac{G_{F}^{2} m E_{\nu}}{2 \pi} \frac{4(1-y)^{2}\left[1-\left(\mu^{2}-m^{2}\right) / 2 m E_{\nu}\right]^{2}}{\left(1-2 m E_{\nu} / M_{W}^{2}\right)^{2}+\Gamma_{W}^{2} / M_{W}^{2}}
\end{aligned}
$$

and

$$
\frac{d \sigma\left(\bar{\nu}_{e} e \rightarrow \text { hadrons }\right)}{d y}=\frac{d \sigma\left(\bar{\nu}_{e} e \rightarrow \bar{\nu}_{\mu} \mu\right)}{d y} \cdot \frac{\Gamma(W \rightarrow \text { hadrons })}{\Gamma\left(W \rightarrow \mu \bar{\nu}_{\mu}\right)}
$$

where $m=0.51099908 \mathrm{MeV} / c^{2}$ is the electron mass and $\mu=105.658389 \mathrm{MeV} / \mathrm{c}^{2}$ is the muon mass [81]. The chiral couplings of the $Z^{0}$ to the electron are $L_{e}=2 \sin ^{2} \theta_{W}-1$ and $R_{e}=2 \sin ^{2} \theta_{W}$, with $\sin ^{2} \theta_{W}=0.226$ [76]. To evaluate the cross sections, we use $M_{W}=80.22 \mathrm{GeV} / c^{2}, M_{Z}=90.188 \mathrm{GeV} / c^{2}$, and 
Fig. 10. Cross sections for neutrino interactions on electron targets. At low energies, from largest to smallest cross section, the processes are (i) $\bar{\nu}_{e} e \rightarrow$ hadrons, (ii) $\nu_{\mu} e \rightarrow \mu \nu_{e}$, (iii) $\nu_{e} e \rightarrow \nu_{e} e$, (iv) $\bar{\nu}_{e} e \rightarrow \bar{\nu}_{\mu} \mu$, (v) $\bar{\nu}_{e} e \rightarrow \bar{\nu}_{e} e$, (vi) $\nu_{\mu} e \rightarrow \nu_{\mu} e$, (vii) $\bar{\nu}_{\mu} e \rightarrow \bar{\nu}_{\mu} e$.

$\Gamma_{W}=2.08 \mathrm{GeV}$. The integrated cross sections

$$
\sigma\left(E_{\nu}\right)=\int_{0}^{1} d y \frac{d \sigma\left(E_{\nu}\right)}{d y}
$$

are plotted in Figure 10. Only in the neighborhood of the intermediate-boson resonance are any of the neutrino-electron processes competitive with the neutrino-nucleon cross sections. The cross sections at the resonance peak, $E_{\nu}^{\text {res }}=M_{W}^{2} / 2 m$, are collected in Table 3 , together with the cross sections for neutrino-nucleon scattering.

We shall consider the effects of the $W^{-}$resonance region, $\left(M_{W}-2 \Gamma_{W}\right)^{2} / 2 m=$ $5.7 \mathrm{PeV} \lesssim E_{\nu} \lesssim\left(M_{W}+2 \Gamma_{W}\right)^{2} / 2 m=7.0 \mathrm{PeV}$, on the attenuation of cosmic $\bar{\nu}_{e}$ in the Earth, through the reaction $\bar{\nu}_{e} e \rightarrow W^{-} \rightarrow$ anything, in $\S 5$. In $\S 8.3$ we project the rate of downward-going $\bar{\nu}_{e} e \rightarrow W^{-} \rightarrow \bar{\nu}_{\mu} \mu$ events for various models of the diffuse neutrino flux from active galactic nuclei. 
Table 3

Integrated cross sections for neutrino-electron and neutrino-nucleon scattering at $E_{\nu}^{\text {res }}=M_{W}^{2} / 2 m=6.3 \times 10^{6} \mathrm{GeV}$.

\begin{tabular}{cc}
\hline Reaction & $\sigma\left[\mathrm{cm}^{2}\right]$ \\
\hline$\nu_{\mu} e \rightarrow \nu_{\mu} e$ & $5.86 \times 10^{-36}$ \\
$\bar{\nu}_{\mu} e \rightarrow \bar{\nu}_{\mu} e$ & $5.16 \times 10^{-36}$ \\
$\nu_{\mu} e \rightarrow \mu \nu_{e}$ & $5.42 \times 10^{-35}$ \\
$\nu_{e} e \rightarrow \nu_{e} e$ & $3.10 \times 10^{-35}$ \\
$\bar{\nu}_{e} e \rightarrow \bar{\nu}_{e} e$ & $5.38 \times 10^{-32}$ \\
$\bar{\nu}_{e} e \rightarrow \bar{\nu}_{\mu} \mu$ & $5.38 \times 10^{-32}$ \\
$\bar{\nu}_{e} e \rightarrow \bar{\nu}_{\tau} \tau$ & $5.38 \times 10^{-32}$ \\
$\bar{\nu}_{e} e \rightarrow$ hadrons & $3.41 \times 10^{-31}$ \\
$\bar{\nu}_{e} e \rightarrow$ anything & $5.02 \times 10^{-31}$ \\
$\nu_{\mu} N \rightarrow \mu \mu^{-}+$anything & $1.43 \times 10^{-33}$ \\
$\nu_{\mu} N \rightarrow \nu_{\mu}+$ anything & $6.04 \times 10^{-34}$ \\
$\bar{\nu}_{\mu} N \rightarrow \mu^{+}+$anything & $1.41 \times 10^{-33}$ \\
$\bar{\nu}_{\mu} N \rightarrow \bar{\nu}_{\mu}+$ anything & $5.98 \times 10^{-34}$ \\
\hline
\end{tabular}

\section{The Earth is Opaque to UHE Neutrinos}

The rise of the charged-current and neutral-current cross sections with energy is mirrored in the decrease of the (water-equivalent) interaction length,

$$
\mathcal{L}_{\text {int }}=\frac{1}{\sigma_{\nu N}\left(E_{\nu}\right) N_{\mathrm{A}}},
$$

where $N_{\mathrm{A}}=6.022 \times 10^{23} \mathrm{~mol}^{-1}=6.022 \times 10^{23} \mathrm{~cm}^{-3}$ (water equivalent) is Avogadro's number. The energy dependence of the interaction lengths for neutrinos on nucleons is shown in Figure 11. We show separately the interaction lengths for charged-current and neutral-current reactions, as well as the interaction length corresponding to the total (charged-current plus neutral-current) cross section. The same information is shown for antineutrinos on nucleons in Figure 12. Above about $10^{16} \mathrm{eV}$, the two sets of interaction lengths coincide. These results apply equally to $\nu_{e} N\left(\right.$ or $\left.\bar{\nu}_{e} N\right)$ collisions as to $\nu_{\mu} N\left(\right.$ or $\left.\bar{\nu}_{\mu} N\right)$ collisions.

Over the energy range of interest for neutrino astronomy, the interactions of $\nu_{e}$, $\nu_{\mu}$, and $\bar{\nu}_{\mu}$ with electrons in the Earth can generally be neglected in compari- 


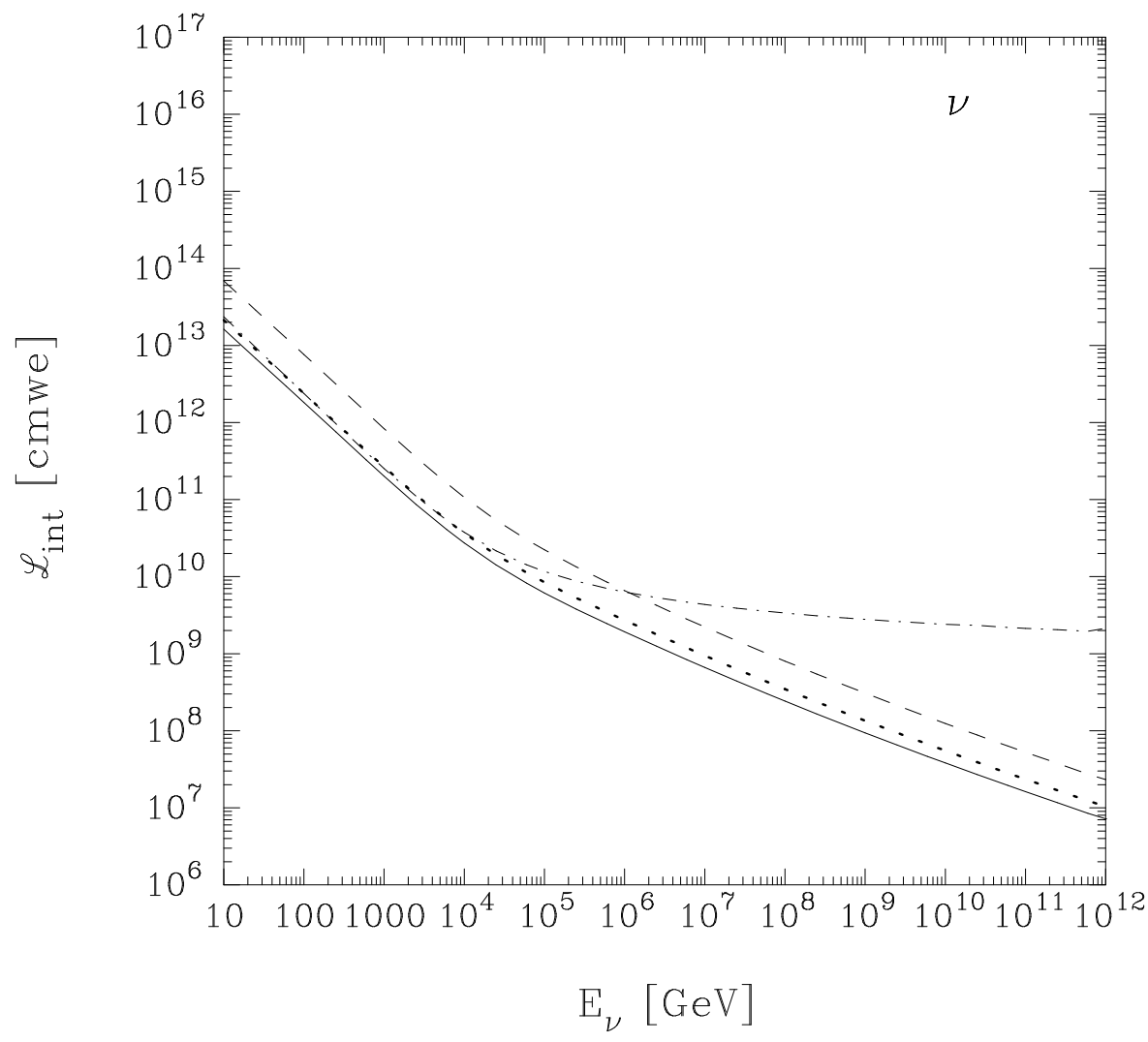

Fig. 11. Interaction lengths for neutrino interactions on nucleon targets: dotted line, charged-current interaction length; dashed line, neutral-current interaction length; solid line, total interaction length, all computed with the CTEQ-DIS parton distributions. The dot-dashed curve shows the charged-current interaction length based on the EHLQ structure functions with $Q^{2}$ fixed at $Q_{0}^{2}=5 \mathrm{GeV}^{2}$, as in Figure 4.

son to interactions with nucleons. The case of $\bar{\nu}_{e} e$ interactions is exceptional, because of the intermediate-boson resonance formed in the neighborhood of $E_{\nu}^{\mathrm{res}}=M_{W}^{2} / 2 m \approx 6.3 \times 10^{15} \mathrm{eV}$. The resonant reactions $\bar{\nu}_{e} e \rightarrow W^{-} \rightarrow \bar{\nu}_{\mu} \mu$ and $\bar{\nu}_{e} e \rightarrow W^{-} \rightarrow$ hadrons may offer a detectable signal. At resonance, the reaction $\bar{\nu}_{e} e \rightarrow W^{-} \rightarrow$ anything significantly attenuates a $\bar{\nu}_{e}$ beam propagating through the Earth. The water-equivalent interaction lengths corresponding to the neutrino-electron cross sections computed in $\S 4$ are displayed in Figure 13. These are evaluated as

$$
\mathcal{L}_{\text {int }}^{(e)}=\frac{1}{\sigma_{\nu e}\left(E_{\nu}\right)(10 / 18) N_{\mathrm{A}}}
$$

where $(10 / 18) N_{\mathrm{A}}$ is the number of electrons in a mole of water.

To good approximation, the Earth may be regarded as a spherically symmetric ball with a complex internal structure consisting of a dense inner and outer core and a lower mantle of medium density, covered by a transition zone, lid, crust, and oceans [82]. A convenient representation of the density profile of 


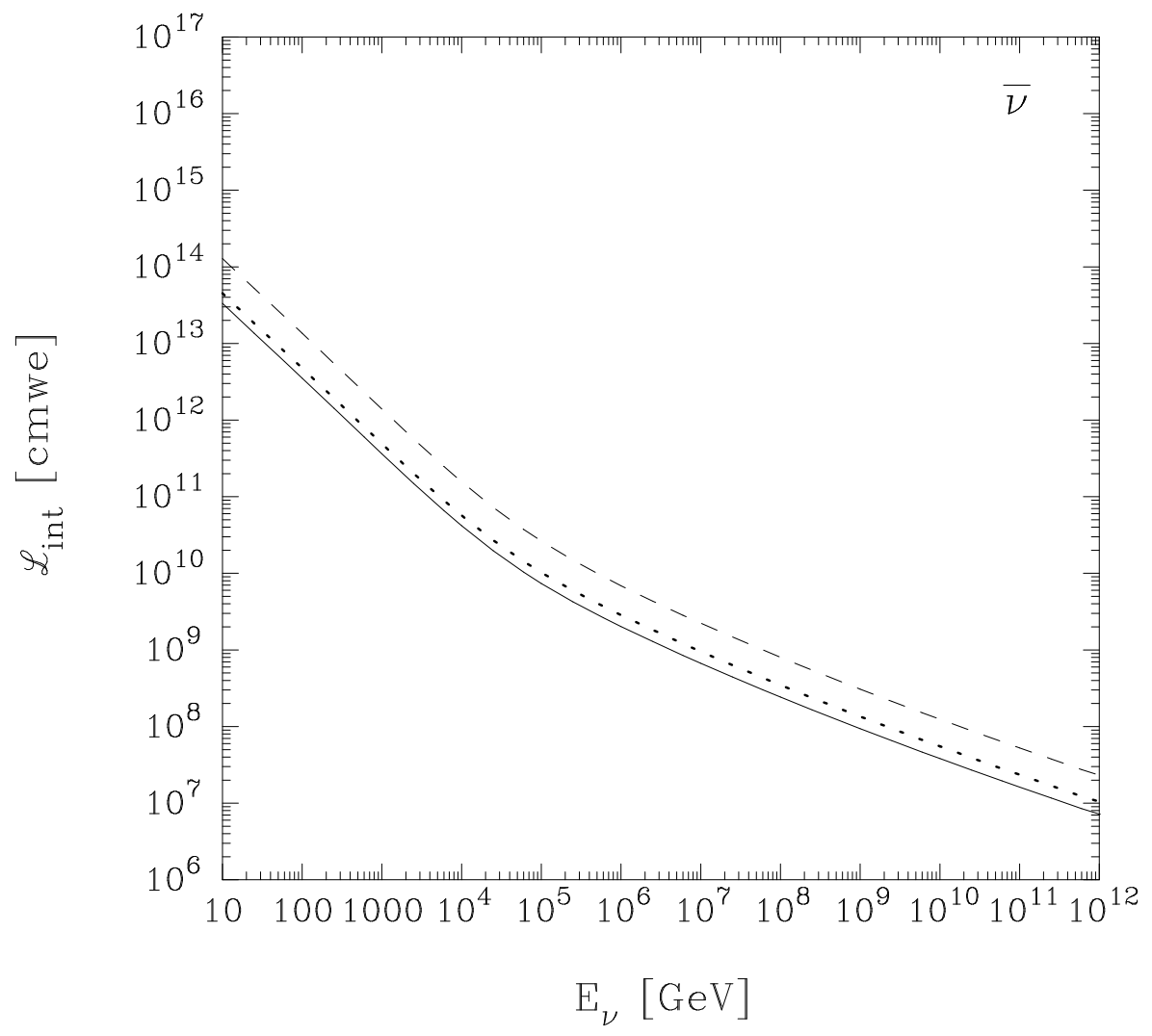

Fig. 12. Interaction lengths for antineutrino interactions on nucleon targets: dotted line, charged-current interaction length; dashed line, neutral-current interaction length; solid line, total interaction length, all computed with the CTEQ-DIS parton distributions.

the Earth is given by the Preliminary Earth Model [83],

$$
\rho(r)= \begin{cases}13.0885-8.8381 x^{2}, & r<1221.5 \\ 12.5815-1.2638 x-3.6426 x^{2}-5.5281 x^{3}, & 1221.5<r<3480 \\ 7.9565-6.4761 x+5.5283 x^{2}-3.0807 x^{3}, & 3480<r<5701 \\ 5.3197-1.4836 x, & 5701<r<5771 \\ 11.2494-8.0298 x, & 5771<r<5971 \\ 7.1089-3.8045 x, & 5971<r<6151 \\ 2.691+0.6924 x, & 6151<r<6346.6 \\ 2.9, & 6346.6<r<6356 \\ 2.6, & 6356<r<6368 \\ 1.02, & r \leq R_{\oplus},\end{cases}
$$




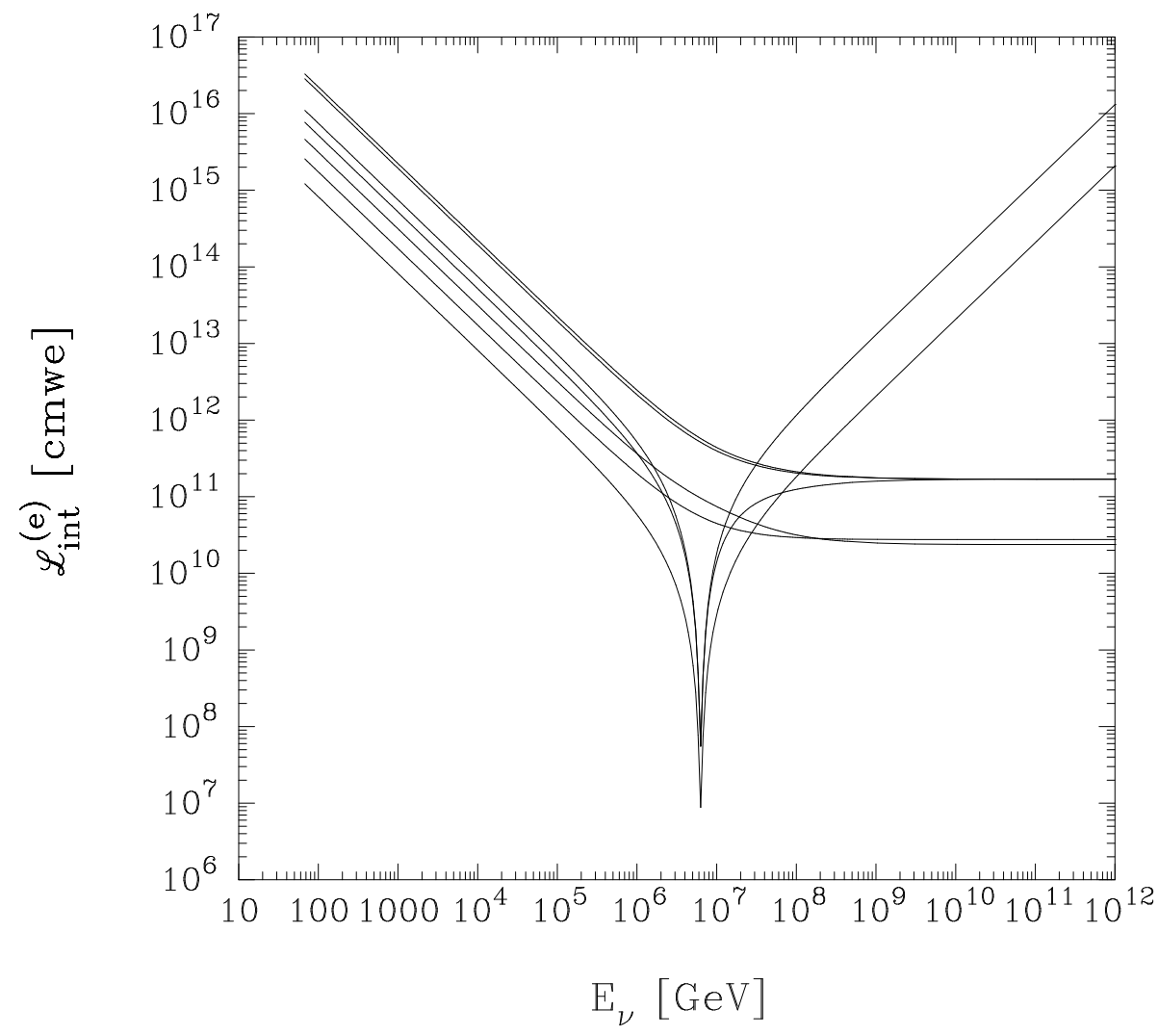

Fig. 13. Interaction lengths for neutrino interactions on electron targets. At low energies, from smallest to largest interaction length, the processes are (i) $\bar{\nu}_{e} e \rightarrow$ hadrons, (ii) $\nu_{\mu} e \rightarrow \mu \nu_{e}$, (iii) $\nu_{e} e \rightarrow \nu_{e} e$, (iv) $\bar{\nu}_{e} e \rightarrow \bar{\nu}_{\mu} \mu$, (v) $\bar{\nu}_{e} e \rightarrow \bar{\nu}_{e} e$, (vi) $\nu_{\mu} e \rightarrow \nu_{\mu} e$, (vii) $\bar{\nu}_{\mu} e \rightarrow \bar{\nu}_{\mu} e$.

where the density is measured in $\mathrm{g} / \mathrm{cm}^{3}$, the distance $r$ from the center of the Earth is measured in $\mathrm{km}$ and the scaled radial variable $x \equiv r / R_{\oplus}$, with the Earth's radius $R_{\oplus}=6371 \mathrm{~km}$. The density of a spherically symmetric Earth is plotted in Figure 14.

The amount of material encountered by an upward-going neutrino in its passage through the Earth is shown in Figure 15 as a function of the neutrino direction. The influence of the core is clearly visible at angles below about $0.2 \pi$. A neutrino emerging from the nadir has traversed a column whose depth is 11 kilotonnes $/ \mathrm{cm}^{2}$, or $1.1 \times 10^{10}$ cmwe. The Earth's diameter exceeds the chargedcurrent interaction length of neutrinos with energy greater than $40 \mathrm{TeV}$. In the interval $2 \times 10^{6} \mathrm{GeV} \lesssim E_{\nu} \lesssim 2 \times 10^{7} \mathrm{GeV}$, resonant $\bar{\nu}_{e} e$ scattering adds dramatically to the attenuation of electron antineutrinos. At resonance, the interaction length due to the reaction $\bar{\nu}_{e} e \rightarrow W^{-} \rightarrow$ anything is 6 tonnes $/ \mathrm{cm}^{2}$, or $6 \times 10^{6}$ cmwe, or 60 kmwe. The resonance is effectively extinguished for neutrinos that traverse the Earth.

We discuss the effect of attenuation on interaction rates of upward-going muonneutrinos in $\S 8.2$. 
Fig. 15. Thickness of the Earth as a function of the angle of incidence of the incoming neutrinos.

\section{UHE Neutrino Interactions in the Atmosphere}

The atmosphere is more than a thousand times less dense than the Earth's interior, so it makes a negligible contribution to the attenuation of the incident neutrino flux. The US Standard Atmosphere (1976) [84] can be reproduced to 
$3 \%$ approximation by the following simple parametrization:

$$
\rho_{\mathrm{atm}}(h)=\left\{\begin{array}{l}
1.225 \times 10^{-3} \mathrm{~g} / \mathrm{cm}^{3} \exp (-h / 9.192 \mathrm{~km}), h<10 \mathrm{~km}, \\
1.944 \times 10^{-3} \mathrm{~g} / \mathrm{cm}^{3} \exp (-h / 6.452 \mathrm{~km}) h \geq 10 \mathrm{~km}
\end{array}\right.
$$

For a standard atmosphere, a neutrino normally incident on a surface detector passes through a column density of $1033 \mathrm{~g} / \mathrm{cm}^{2}=1033 \mathrm{cmwe}$, while a neutrino arriving along the horizon passes through a column of about 36000 cmwe. Both amounts of matter are orders of magnitude smaller than the neutrino interaction lengths at the energies under study (cf. Figures 11, 12, and 13). The atmosphere is thus essentially transparent to neutrinos.

On the other hand, the amount of material encountered by a neutrino passing horizontally through the atmosphere is not small compared with the depth available for the production of contained events in a water (or ice) Cerenkov detector. Figure 16 shows the column depth traversed by a horizontal neutrino as a function of altitude. (The values shown are for the full passage through the atmosphere, not just inbound to the point of closest approach to the surface.) An air shower detector like the Fly's Eye [85], which detects light produced by nitrogen fluorescence along the path of a high-energy particle traversing the atmosphere, could detect neutrino-induced cascades and perhaps identify their shower profiles. Indeed, Halzen, et al. [86] have argued that the $3 \times 10^{20}-\mathrm{eV}$ cosmic ray shower observed by Fly's Eye [87], the highest energy cosmic-ray event, might have been initiated by a neutrino. Sigl and Lee [88] comment that the interpretation of the highest-energy cosmic rays as neutrino interactions in the atmosphere becomes likely only if $\sigma_{\mathrm{CC}}(\nu N)$ were a few orders of magnitude higher than we calculate.

\section{Shadows of the Moon and Sun}

In recent years, cosmic-ray experiments have used the observation of shadowing of the cosmic-ray flux by the Moon and Sun to demonstrate the angular resolution of their detectors [89]. Might it someday be possible to observe the shadowing of neutrinos by Earth's satellite and star?

The Moon has a radius of $R_{\text {Moon }}=1738 \mathrm{~km}$ and an average density of $\left\langle\rho_{\text {Moon }}\right\rangle=3.37 \mathrm{~g} / \mathrm{cm}^{3}$. It is approximately uniform in density, except for a

core at $R<238 \mathrm{~km}$, where $\rho_{\text {Moon }} \approx 7.55 \mathrm{~g} / \mathrm{cm}^{3}$ [90]. The column depth along the lunar diameter is $1.378 \times 10^{9}$ cmwe, which makes the Moon opaque to neutrinos with $E_{\nu} \gtrsim 10^{6} \mathrm{GeV}$.

The matter distribution in the Sun extends to a solar radius of $R_{\odot}=6.96 \times$ 


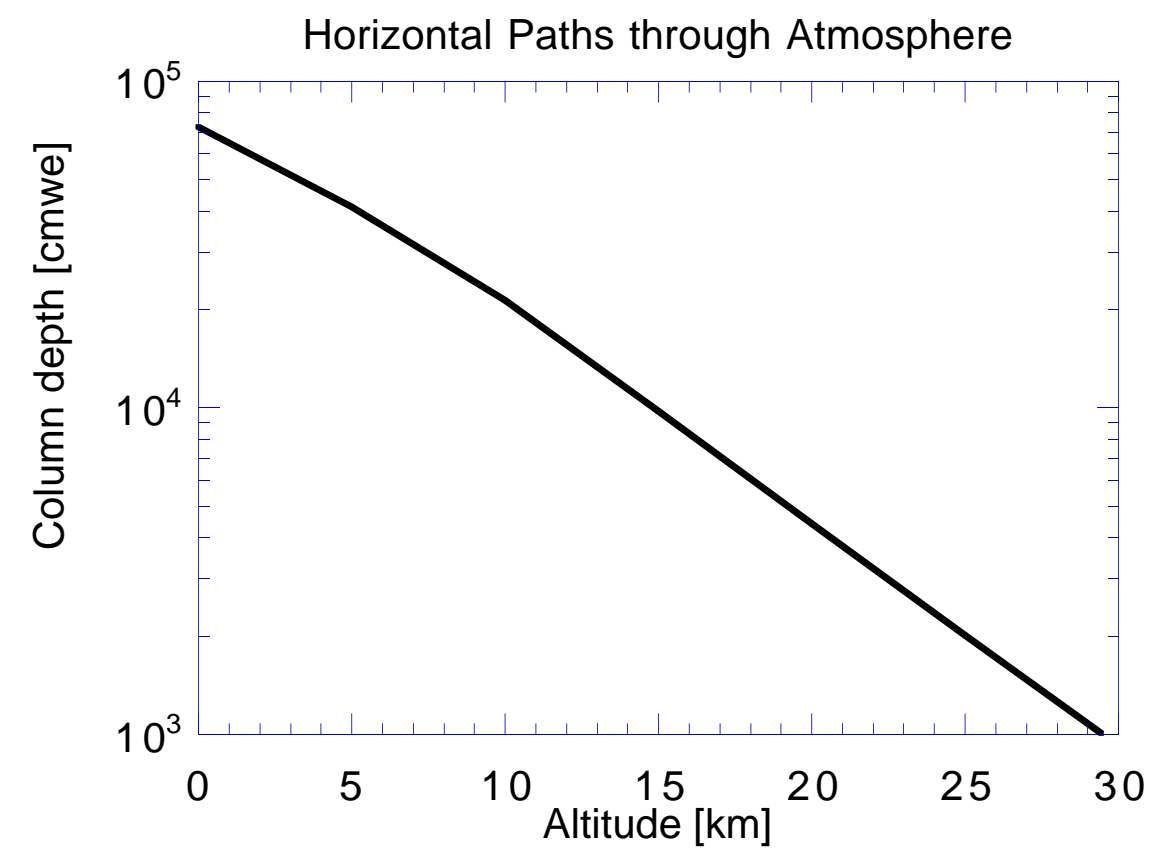

Fig. 16. Column depth encountered by a horizontal neutrino traversing Earth's atmosphere at an altitude $h$.

$10^{5} \mathrm{~km}$. The density distribution is known from the standard solar model [91]. Except very near the center, a good description is given by the simple parametrization,

$$
\rho_{\odot}=236.93 \mathrm{~g} / \mathrm{cm}^{3} \exp \left(-10.098 \mathrm{r} / R_{\odot}\right) .
$$

The profile through the solar diameter is $3.27 \times 10^{12}$ cmwe, which makes the Sun opaque to neutrinos with $E_{\nu} \gtrsim 100 \mathrm{GeV}$. The column density encountered by parallel rays of neutrinos falling on the Sun is shown as a function of distance from the center of the Sun's face in Figure 17. Almost the entire face of the Sun is opaque to neutrinos with energies above $10^{6} \mathrm{GeV}$.

Since the Moon and Sun are small in the sky, each with an angular diameter of about $1 / 2^{\circ}$, both large detector volumes and excellent angular resolution will be required to see their shadows.

\section{UHE Neutrino Fluxes and Event Rates}

In this section, we calculate event rates for atmospheric neutrinos, cosmic neutrinos and neutrinos that originate in active galactic nuclei. We start with a brief discussion of theoretical models for UHE neutrino production and their predictions for the energy dependence of muon-neutrino and electron-neutrino fluxes. We consider representative fluxes in order to assess the feasibility of 


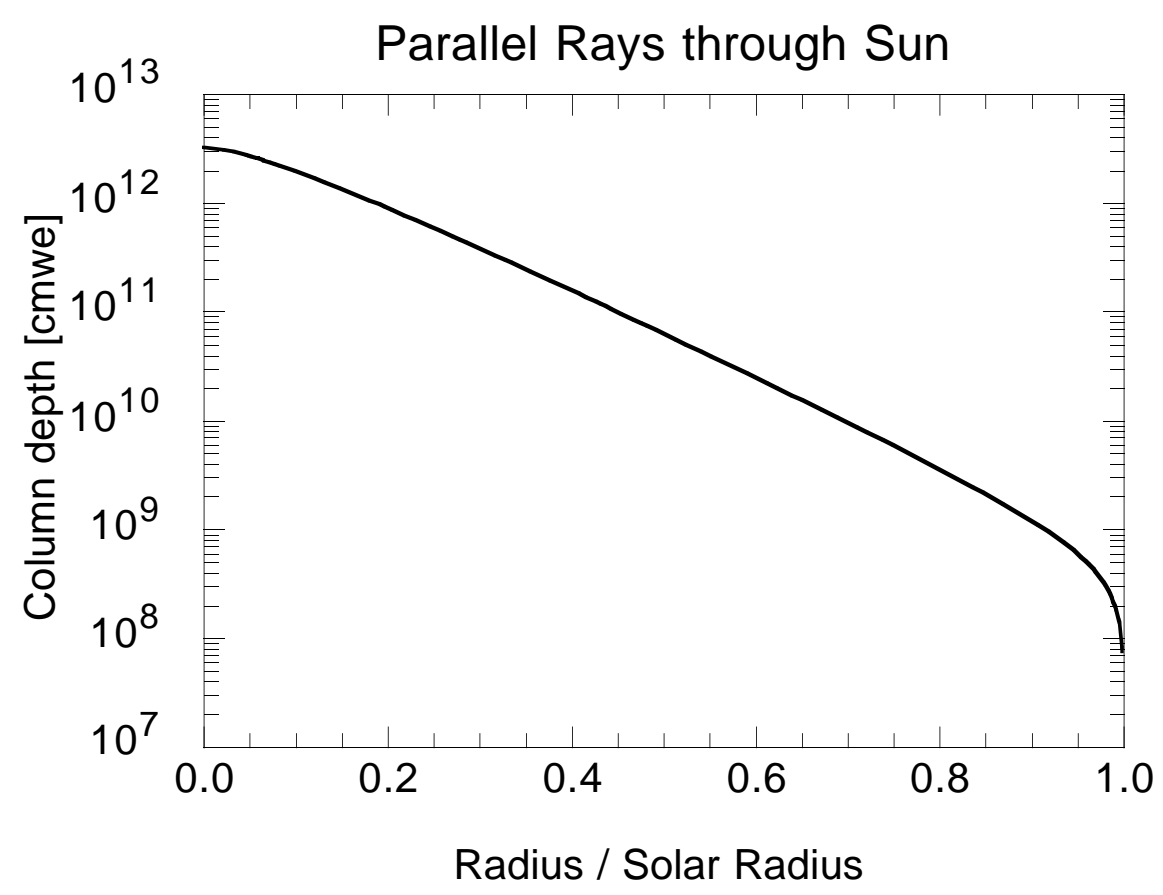

Fig. 17. Column depth presented by the Sun to parallel streams of neutrinos.

detection and examine the consequences of our new neutrino-nucleon cross sections. We first calculate the event rates for upward-moving muons and antimuons produced in the material below the detector, and then consider rates for downward-moving and contained events for both muon- and electronneutrino interactions.

\subsection{Sources of UHE Neutrinos}

In Figures 18 and 19 we display differential neutrino fluxes from a variety of sources. Neutrinos produced by cosmic-ray interactions in the atmosphere dominate other neutrino sources at energies below $1 \mathrm{TeV}$. For the detection of extraterrestrial neutrinos we focus on neutrino energies above $1 \mathrm{TeV}$. The solid curves shown in Figure 18 represent $\nu_{\mu}+\bar{\nu}_{\mu}$ fluxes produced by several mechanisms, while Figure 19 shows the $\nu_{e}+\bar{\nu}_{e}$ fluxes.

The "conventional" atmospheric neutrino flux at $E_{\nu}=1 \mathrm{TeV}$ is derived from the decay of charged pions and kaons produced by cosmic ray interactions in the atmosphere. The conventional flux calculated by Volkova [92], labeled by ATM in the figures, is exhibited as the angle average of the atmospheric

$\nu_{\mu}+\bar{\nu}_{\mu}$ (Figure 18) and $\nu_{e}+\bar{\nu}_{e}$ (Figure 19) fluxes. The predicted horizontal neutrino spectra are in agreement with the absolute spectra measured in the Fréjus experiment up to $10 \mathrm{TeV}[12,93]$. The atmospheric neutrino flux is large at $E_{\nu}=1 \mathrm{TeV}$, but the spectrum falls rapidly as a function of energy. For 


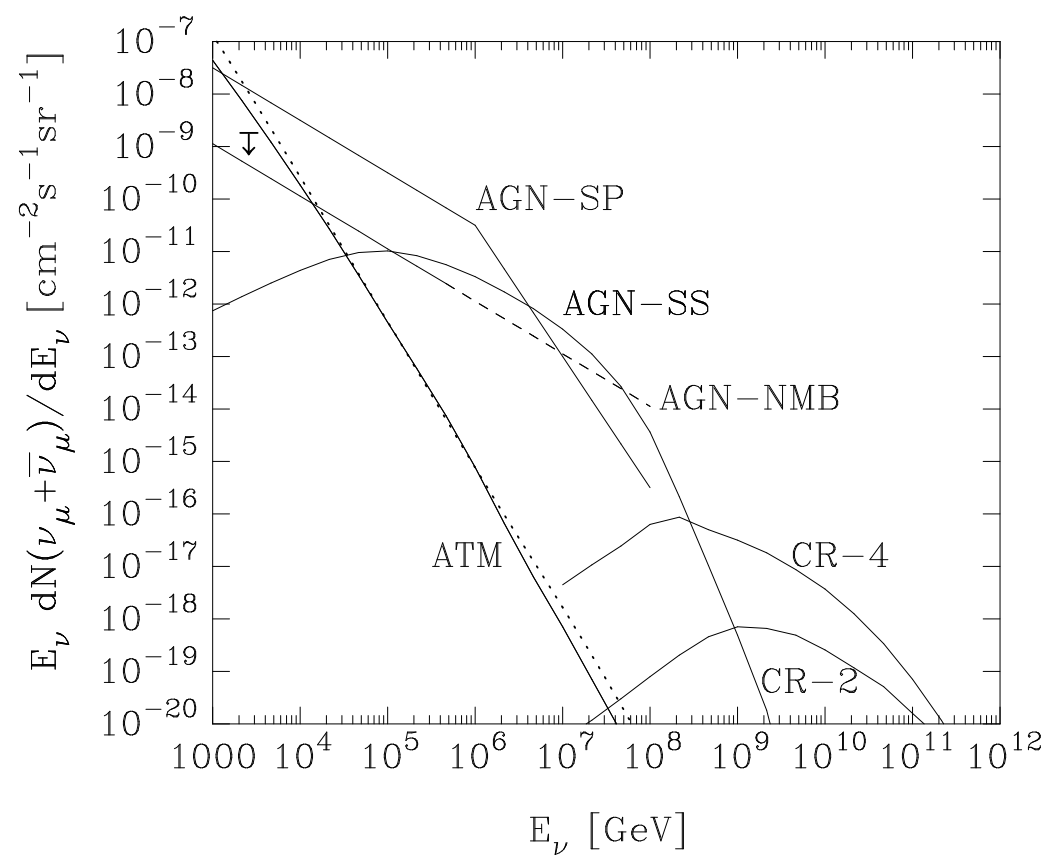

Fig. 18. Muon neutrino plus antineutrino fluxes at the Earth's surface: angle-averaged flux from cosmic-ray interactions in the atmosphere (ATM), and isotropic fluxes from active galactic nuclei (AGN-SS, AGN-NMB, and AGN-SP) and from cosmic-ray interactions with the microwave background (CR-2 and CR-4). The Fréjus upper limit [101] for a neutrino flux in excess of the atmospheric neutrino flux is indicated at $2.6 \mathrm{TeV}$. The dotted line shows the vertical flux of atmospheric $\mu^{+}+\mu^{-}$calculated in Ref. [94].

$1 \mathrm{TeV}<E_{\nu}<10^{3} \mathrm{TeV}$, the angle-averaged atmospheric $\nu_{\mu}+\bar{\nu}_{\mu}$ flux can be approximated by a power law spectrum:

$$
\frac{d N_{\nu_{\mu}+\bar{\nu}_{\mu}}}{d E_{\nu}}=7.8 \times 10^{-11}\left(\frac{E_{\nu}}{1 \mathrm{TeV}}\right)^{-3.6} \mathrm{~cm}^{-2} \mathrm{~s}^{-1} \mathrm{sr}^{-1} \mathrm{GeV}^{-1}
$$

The use of the angle-averaged atmospheric flux, while not necessary, facilitates comparison with fluxes from diffuse extraterrestrial sources.

An additional "prompt" contribution to the atmospheric flux arises from charm production and decay. The vertical prompt neutrino flux has recently been reëxamined using the Lund model for particle production [94], and has been shown to be small relative to the conventional atmospheric flux for $E_{\nu}<10^{5} \mathrm{GeV}$. Since atmospheric neutrinos are a significant background only for $E_{\nu} \lesssim 10 \mathrm{TeV}$, we neglect neutrinos from charm decay in our calculations of event rates.

We also show in Figure 18 the vertical atmospheric muon flux from conventional and prompt sources [94], indicated by a dotted line. The atmospheric muon flux for $E_{\mu}>10^{7} \mathrm{GeV}$ is dominated by muons from charm decays. The 


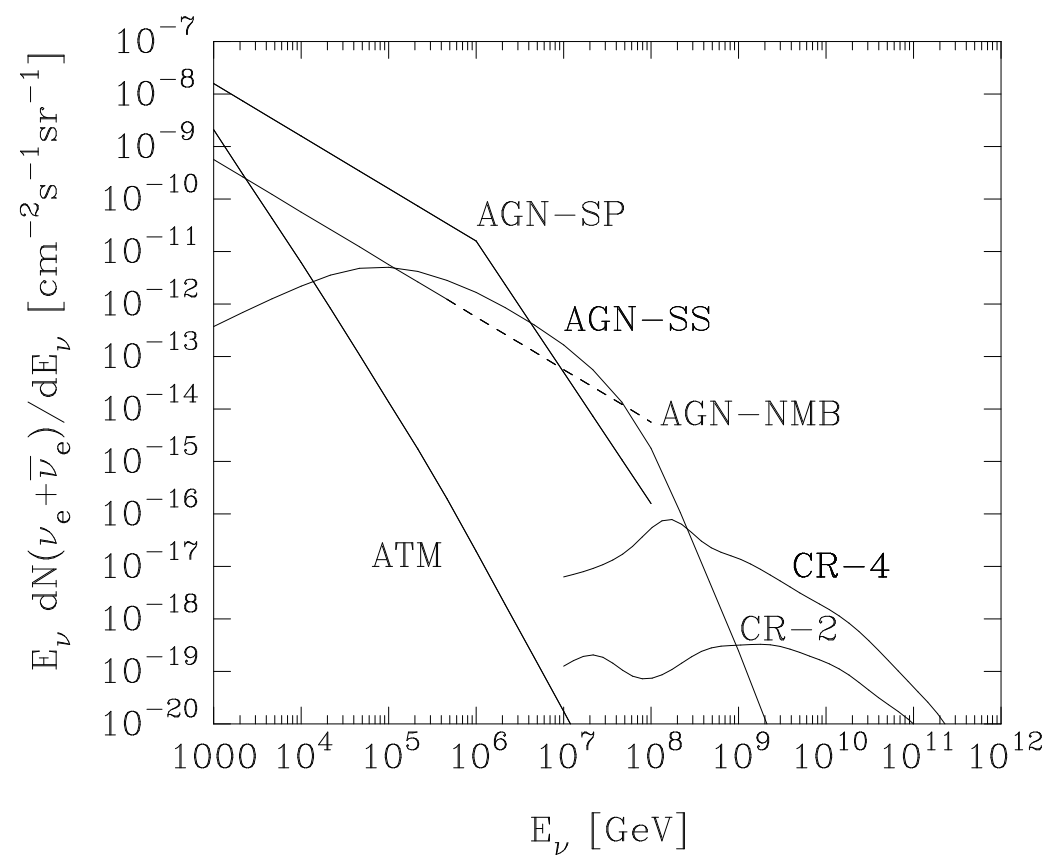

Fig. 19. Electron neutrino plus antineutrino fluxes at the Earth's surface: angle-averaged flux from cosmic-ray interactions in the atmosphere (ATM), and isotropic fluxes from active galactic nuclei (AGN-SS, AGN-NMB, and AGN-SP) and from cosmic-ray interactions with the microwave background (CR-2 and CR-4).

muon spectrum at sea level is approximately parametrized by

$$
\frac{d N_{\mu+\bar{\mu}}}{d E_{\mu}}=1.05 \times 10^{-10}\left(\frac{E_{\mu}}{1 \mathrm{TeV}}\right)^{-3.7} \mathrm{~cm}^{-2} \mathrm{~s}^{-1} \mathrm{sr}^{-1} \mathrm{GeV}^{-1}
$$

Atmospheric muons from charm decay and from conventional sources constitute a background to the detection of $\nu_{\mu} N$ charged-current interactions. By deploying a detector at great depths [95], or observing upward-going muons, or both, one can reduce the cosmic-ray muons to a manageable background.

Detectable fluxes of neutrinos may be generated in active galactic nuclei [96]. The observation [97] that the diffuse neutrino flux from unresolved AGNs might be observable with the proposed neutrino telescopes has stimulated a number of calculations of the diffuse UHE neutrino and cosmic-ray fluxes due to AGNs. Many models for the isotropic neutrino flux from the sum of all AGN sources appear in the literature [98]. We consider three models as representative. The flux calculated by Stecker and Salamon [97], labelled AGN-SS in Figures 18 and 19, has significant contributions from $p p$ and $p \gamma$ interactions in the accretion disk. In the model of Nellen, Mannheim, and Bierman [99], labelled AGN-NMB, $p p$ collisions are the dominant neutrino source, leading 
to a flux

$$
\frac{d N_{\nu_{\mu}+\bar{\nu}_{\mu}}}{d E_{\nu}}=1.13 \times 10^{-12}\left(\frac{E_{\nu}}{1 \mathrm{TeV}}\right)^{-2} \mathrm{~cm}^{-2} \mathrm{~s}^{-1} \mathrm{sr}^{-1} \mathrm{GeV}^{-1}
$$

for $E_{\nu} \lesssim 4 \times 10^{5} \mathrm{GeV}$. At higher energies one expects the spectrum to steepen, because of the lack of parent protons to produce neutrinos. In our rate estimates, we use the analytic form (30) up to $E_{\nu}=10^{8} \mathrm{GeV}$ and comment on the effect of truncating the neutrino energy spectrum. Szabo and Protheroe [100] have extended the model of Stecker and collaborators to include all the important energy-loss mechanisms and computed neutrino production in radio-quiet AGNs and in the central regions of radio-loud AGNs. Their model results in significantly higher fluxes in the energy range between $1 \mathrm{TeV}$ and $10^{3} \mathrm{TeV}$. We take the parametrization

$$
\frac{d N_{\nu_{\mu}+\bar{\nu}_{\mu}}}{d E_{\nu}}=\left\{\begin{array}{l}
10^{-10.5}\left(\frac{E_{\nu}}{1 \mathrm{TeV}}\right)^{-2} \mathrm{~cm}^{-2} \mathrm{~s}^{-1} \mathrm{sr}^{-1} \mathrm{GeV}^{-1}, E_{\nu} \lesssim 10^{3} \mathrm{TeV}, \\
10^{-6}\left(\frac{E_{\nu}}{1 \mathrm{TeV}}\right)^{-3.5} \mathrm{~cm}^{-2} \mathrm{~s}^{-1} \mathrm{sr}^{-1} \mathrm{GeV}^{-1}, E_{\nu} \gtrsim 10^{3} \mathrm{TeV}
\end{array}\right.
$$

to represent their hardest spectrum, corresponding to a scaled diffusion coefficient, $b=1$. In the interval $1 \mathrm{TeV} \lesssim E_{\nu} \lesssim 10 \mathrm{TeV}$, this flux is in conflict with the upper limit determined by the Fréjus Collaboration [101]. This curve is labelled AGN-SP. The electron-neutrino fluxes are taken to be one-half of the muon-neutrino fluxes.

All of these fluxes are consistent with the upper limits deduced from horizontal air showers by the EAS-TOP Collaboration at Campo Imperatore [102]. For $10^{5} \mathrm{GeV}<E_{\nu}<10^{6} \mathrm{GeV}$, they infer the "all-flavor" bound $\left(\nu=\nu_{e}, \bar{\nu}_{e}, \nu_{\mu}, \bar{\nu}_{\mu}\right)$

$$
\int_{10^{5} \mathrm{GeV}}^{10^{6} \mathrm{GeV}} d E_{\nu} \frac{d N_{\nu}}{d E_{\nu}}<1.5 \times 10^{-8} \mathrm{~cm}^{-2} \mathrm{~s}^{-1} \mathrm{sr}^{-1} .
$$

Assuming that the spectrum in this interval is proportional to $E^{-2}$, they obtain a bound on the differential flux,

$$
\frac{d N_{\nu}}{d E_{\nu}}<1.5 \times 10^{-9}\left(\frac{E_{\nu}}{1 \mathrm{TeV}}\right)^{-2} \mathrm{~cm}^{-2} \mathrm{~s}^{-1} \mathrm{sr}^{-1} \mathrm{GeV}^{-1} .
$$

At the $\bar{\nu}_{e} e \rightarrow W^{-}$resonance energy, the limit on the $\bar{\nu}_{e}$ flux is

$$
\frac{d N_{\bar{\nu}_{e}}}{d E_{\bar{\nu}_{e}}}<7.6 \times 10^{-18} \mathrm{~cm}^{-2} \mathrm{~s}^{-1} \mathrm{sr}^{-1} \mathrm{GeV}^{-1}
$$


The remaining curves represent fluxes from two models of neutrino production in interactions of cosmic rays with the microwave background photons. These fluxes, calculated numerically by Yoshida and Teshima by Monte Carlo methods [103], update earlier analytical results [104,105]. The fluxes depend on the redshifts of the cosmic-ray sources: the CR-4 flux corresponds to a maximum redshift, or turn-on time, of $z_{\max }=4$ and evolution parameter $m=4$, while the CR-2 curve corresponds to $z_{\max }=2$ and $m=0$. The two models represent the extremes presented by Yoshida and Teshima. Separate calculations were made for electron and muon neutrinos.

\section{2 $\nu_{\mu}$ and $\bar{\nu}_{\mu}$ Interactions}

With these representative fluxes, we turn to the calculation of event rates and the implications of the new cross sections presented in $\S 3$. As we have noted in $\S 1$, the effective volume of a detector may be considerably enhanced over

the instrumented volume by recording charged-current $\nu_{\mu} N$ interactions that occur in the rock or ice surrounding the detector. The upward muon event rate is shielded from the flux of atmospheric muons, and has the advantage of utilizing more underground target material. Muons produced with $E_{\mu}=$ $10 \mathrm{TeV}$ will travel, on average, a few kilometers as their energy is degraded to $1 \mathrm{TeV}$. The upward muon event rate depends on the $\nu_{\mu} N$ cross section in two ways: through the interaction length which governs the attenuation of the neutrino flux due to interactions in the Earth, and through the probability that the neutrino converts to a muon energetic enough to arrive at the detector with $E_{\mu}$ larger than the threshold energy $E_{\mu}^{\mathrm{min}}$.

For the case of isotropic fluxes, such as the AGN and cosmic neutrino fluxes presented in $\S 8.1$, the attenuation can be represented by a shadow factor that is equivalent to the effective solid angle for upward muons, divided by $2 \pi$ :

$$
S\left(E_{\nu}\right)=\frac{1}{2 \pi} \int_{-1}^{0} d \cos \theta \int d \phi \exp \left[-z(\theta) / \mathcal{L}_{\text {int }}\left(E_{\nu}\right)\right]
$$

The interaction length $\mathcal{L}_{\text {int }}\left(E_{\nu}\right)$ is shown in Figures 11 and 12 for $\nu N$ and $\bar{\nu} N$ interactions, respectively. The column depth $z(\theta)$ is plotted in Figure 15. We show the shadow factors computed with the CTEQ-DIS, D_ and CTEQDLA total cross sections in Figure 20. All of these lead to greater shadowing than the EHLQ-DLA distributions used in earlier work. In fact, neither the charged-current cross section nor the total cross section is quite appropriate in the shadow factor. Neutral-current interactions degrade the neutrino energy, but do not remove neutrinos from the beam. A full accounting of the effect of neutral currents on the underground upward neutrino flux has been given 


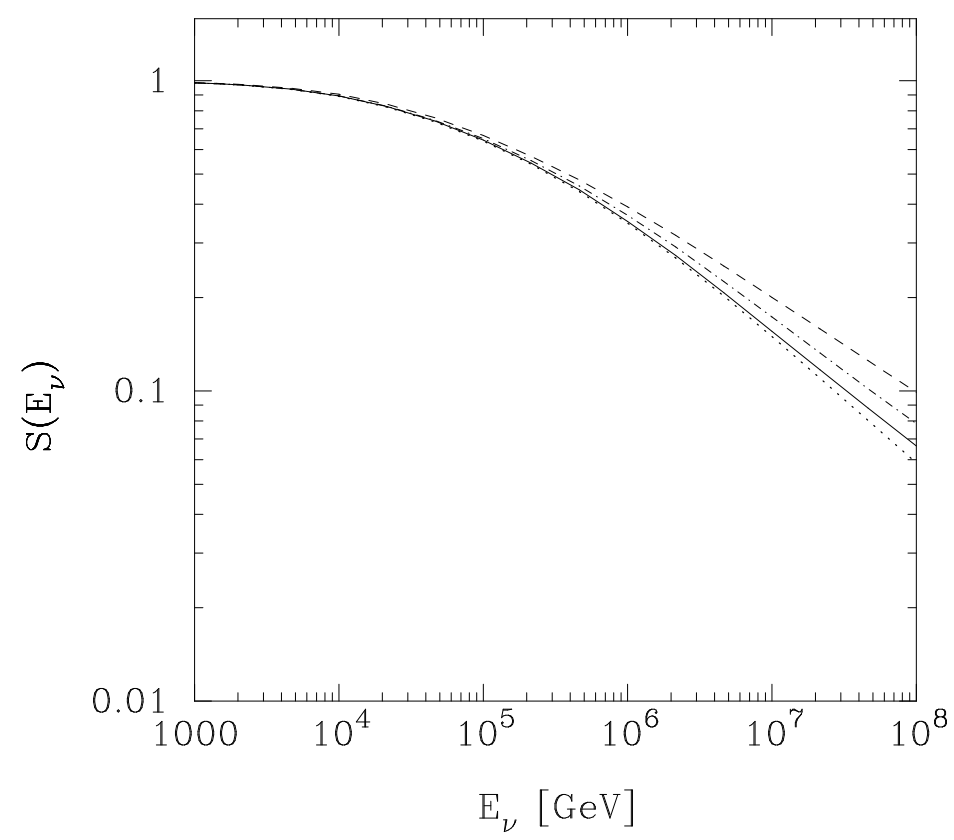

Fig. 20. The shadow factor $S\left(E_{\nu}\right)$ for upward-going neutrinos assuming that $\sigma=\sigma_{\text {tot }}$ in (35) for CTEQ-DIS (solid line), CTEQ-DLA (dot-dashed) and D_ (dotted) parton distribution functions. Also shown is the shadow factor using the EHLQ cross sections (dashed line).

in Ref. [105]. We compute the shadow factor using the interaction lengths for the charged-current and charged-current plus neutral-current interactions to bracket the number of events for a given model. The longer charged-current interaction length leads to higher event rates.

The probability that a muon produced in a charged-current interaction arrives in a detector with an energy above the muon energy threshold $E_{\mu}^{\text {min }}$ depends on the average range $\langle R\rangle$ of a muon in rock,

$$
\left\langle R\left(E_{\nu} ; E_{\mu}^{\min }\right)\right\rangle=\frac{1}{\sigma_{\mathrm{CC}}\left(E_{\nu}\right)} \int_{0}^{1-E_{\mu}^{\min } / E_{\nu}} d y R\left(E_{\nu}(1-y), E_{\mu}^{\min }\right) \frac{d \sigma_{\mathrm{CC}}\left(E_{\nu}, y\right)}{d y}
$$

The range $R$ of an energetic muon follows from the energy-loss relation

$$
-d E_{\mu} / d x=a\left(E_{\mu}\right)+b\left(E_{\mu}\right) E_{\mu} .
$$

If the coefficients $a$ and $b$ are independent of energy, then

$$
R\left(E_{\mu}, E_{\mu}^{\mathrm{min}}\right)=\frac{1}{b} \ln \frac{a+b E_{\mu}}{a+b E_{\mu}^{\min }} .
$$

In our calculations below, we use $a=2.0 \times 10^{-3} \mathrm{GeV}_{\text {cmwe }}{ }^{-1}$ and $b=3.9 \times$ 


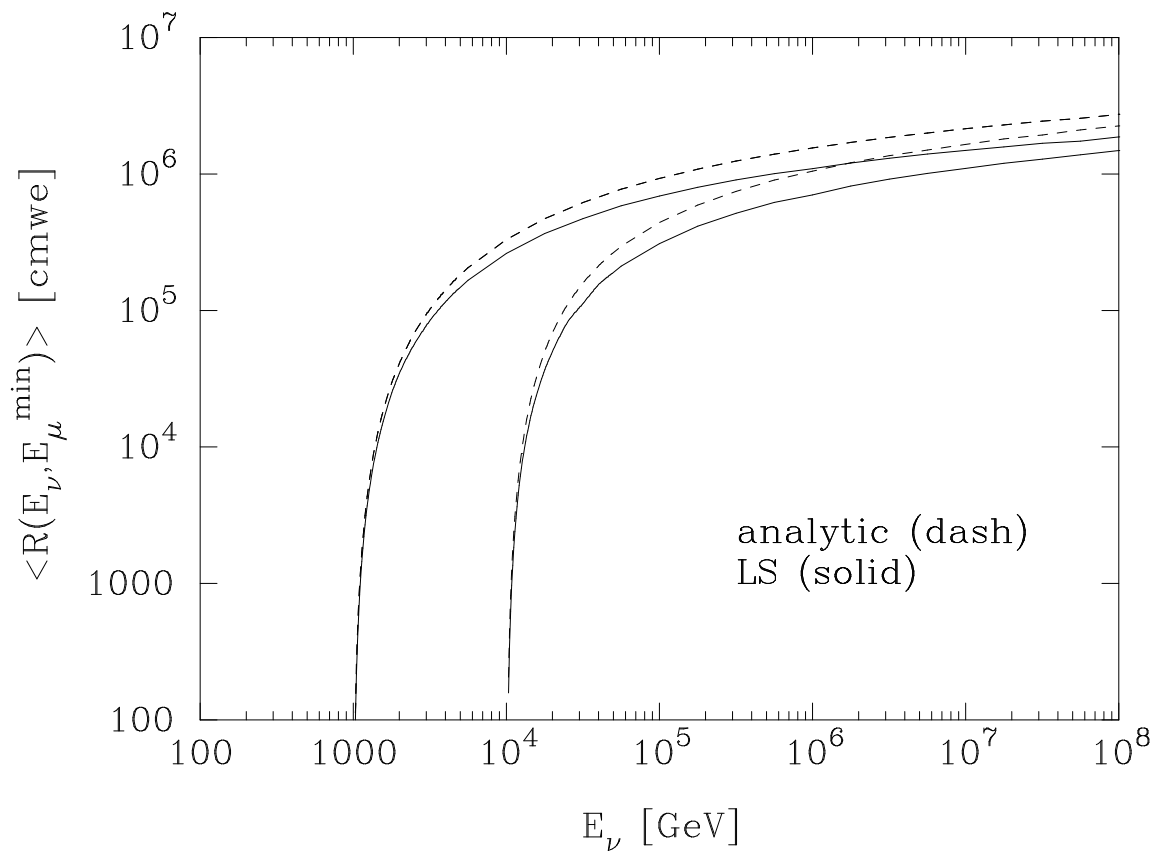

Fig. 21. Mean ranges in rock on muons produced in charged-current interactions of neutrinos with energy $E_{\nu}$. The Lipari-Stanev (solid) and analytic (dashed) ranges are shown for $E_{\mu}^{\min }=1$ and $10 \mathrm{TeV}$.

$10^{-6} \mathrm{cmwe}^{-1}$ in this analytic range formula [106]. We have also considered muon ranges evaluated numerically by Lipari and Stanev, which include the energy dependence in $a$ and $b$ [107]. In Figure 21, we compare the LipariStanev (LS) range and the analytic range for $E_{\mu}^{\min }=1$ and $10 \mathrm{TeV}$. The average range is essentially independent of the parton distribution functions, as they all have the same general form for $d \sigma / d y$.

The probability that a neutrino of energy $E_{\nu}$ produces an observable muon is

$$
P_{\mu}\left(E_{\nu}, E_{\mu}^{\min }\right)=N_{A} \sigma_{\mathrm{CC}}\left(E_{\nu}\right)\left\langle R\left(E_{\nu} ; E_{\mu}^{\min }\right)\right\rangle
$$

where $N_{A}$ is Avogadro's number. The event rate for a detector with effective area $A$ is

$$
\text { Rate }=A \int d E_{\nu} P_{\mu}\left(E_{\nu} ; E_{\mu}^{\min }\right) S\left(E_{\nu}\right) \frac{d N}{d E_{\nu}}
$$

The $\nu_{\mu} \rightarrow \mu$ probabilities are plotted in Figure 22 for the three new parton distribution functions, as well as the EHLQ-DLA parton distributions, for $E_{\mu}^{\min }=1$ and $10 \mathrm{TeV}$. The effect of the larger cross sections is to increase the probability that a neutrino produces an observable muon, but also to increase the attenuation of neutrinos en route to the detector. The net effect is that for the CTEQ-DIS, CTEQ-DLA and D_ cross sections, the combination $P_{\mu}\left(E_{\nu}, E_{\mu}^{\min }\right) S\left(E_{\nu}\right)$ has little dependence on the choice of parton distribution 


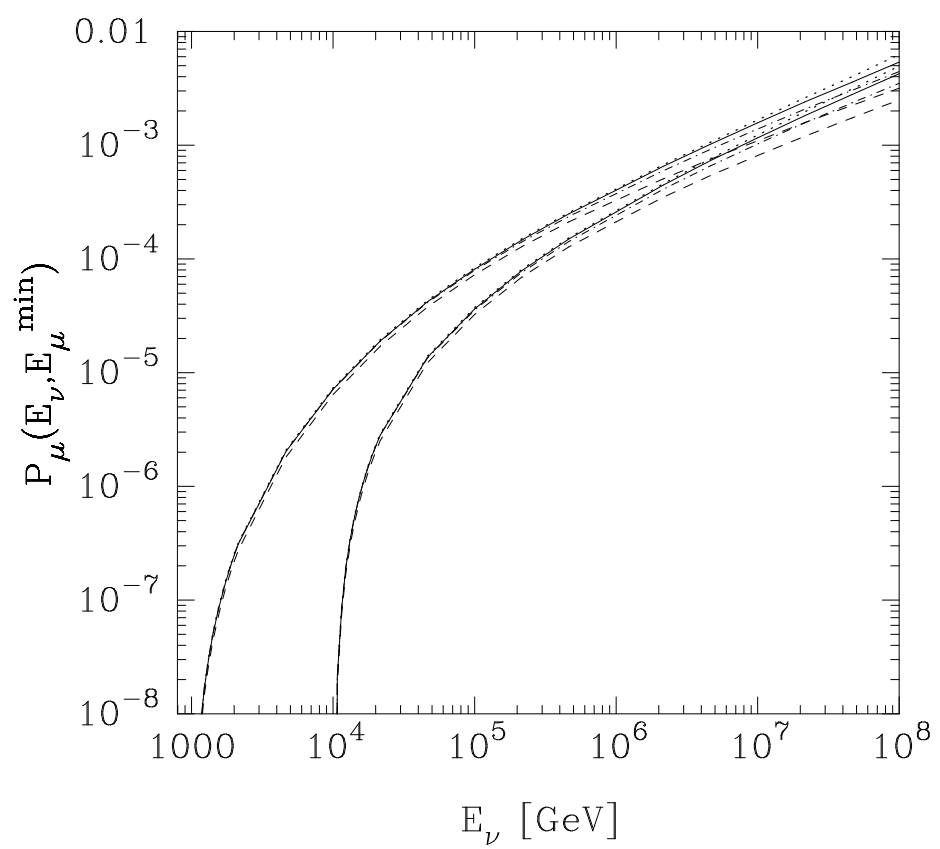

Fig. 22. Probability that a neutrino of energy $E_{\nu}$ produces an observable muon with energy exceeding $E_{\mu}^{\mathrm{min}}=1$ and $10 \mathrm{TeV}$, calculated for the Lipari-Stanev range. The curves correspond to the CTEQ-DIS (solid), CTEQ-DLA (dot-dashed), D_ (dotted), and EHLQ-DLA (dashed) parton distributions.

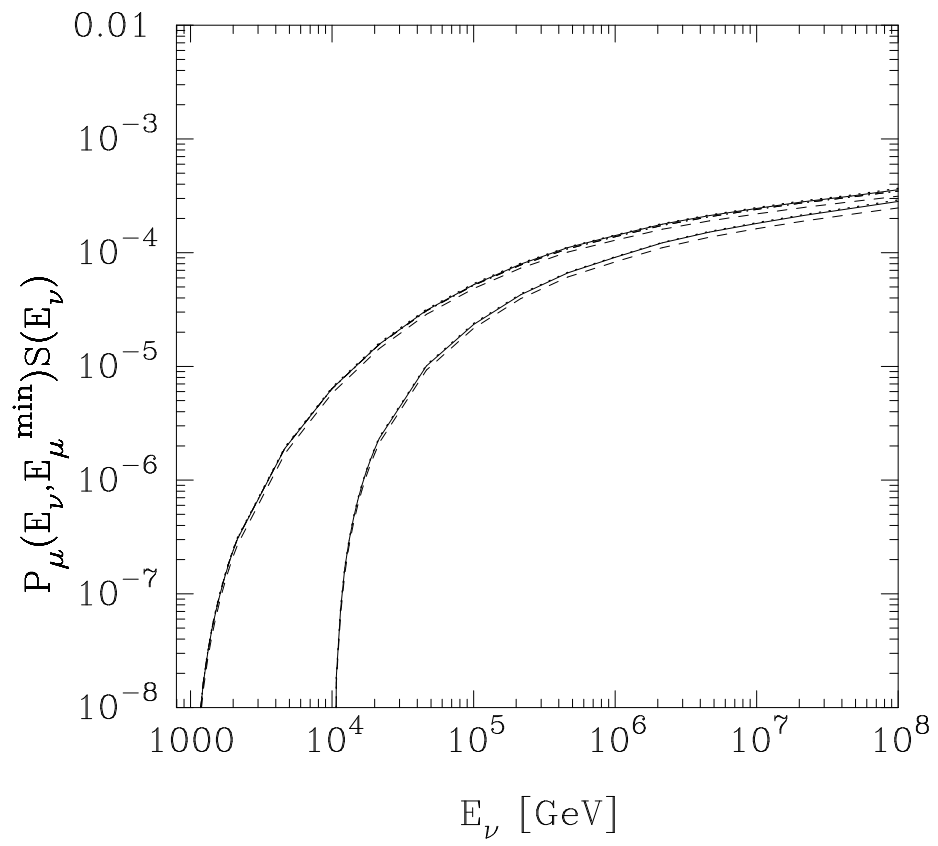

Fig. 23. The product $P_{\mu}\left(E_{\nu}, E_{\mu}^{\min }\right) S\left(E_{\nu}\right)$, calculated using the Lipari-Stanev range and shadow factor determined by the total cross section, for $E_{\mu}^{\min }=1$ and $10 \mathrm{TeV}$. The curves correspond to the CTEQ-DIS (solid), CTEQ-DLA (dot-dashed), D_ (dotted), and EHLQ-DLA (dashed) parton distributions.

functions, as seen in Figure 23. The CTEQ-DLA and D_ distributions yield upward event rates within a few percent of those calculated for CTEQ-DIS dis- 
Table 4

Upward $\mu^{+}+\mu^{-}$event rates per steradian per year arising from $\nu_{\mu} N$ and $\bar{\nu}_{\mu} N$ interactions in rock, for a detector with effective area $A=0.1 \mathrm{~km}^{2}$ and muon energy threshold $E_{\mu}^{\min }=1 \mathrm{TeV}$. The smaller value of each pair corresponds to attenuation by the total cross section; the larger to attenuation by charged-current interactions.

\begin{tabular}{cccc}
\multirow{2}{*}{ Flux } & \multicolumn{2}{c}{ CTEQ-DIS } & EHLQ-DLA \\
& analytic & LS & LS \\
\hline ATM [92] & $170-173$ & $138-141$ & $124-126$ \\
AGN-SS [97] & $106-126$ & $77-92$ & $70-82$ \\
AGN-NMB [99] & $134-146$ & $102-111$ & $93-100$ \\
AGN-SP [100] & $3570-3870$ & $2740-2960$ & $2440-2660$ \\
\hline
\end{tabular}

Table 5

Upward $\mu^{+}+\mu^{-}$event rates per steradian per year arising from $\nu_{\mu} N$ and $\bar{\nu}_{\mu} N$ interactions in rock, for a detector with effective area $A=0.1 \mathrm{~km}^{2}$ and muon energy threshold $E_{\mu}^{\min }=10 \mathrm{TeV}$. The smaller value of each pair corresponds to attenuation by the total cross section; the larger to attenuation by charged-current interactions.

\begin{tabular}{cccc}
\hline \multirow{2}{*}{ Flux } & \multicolumn{2}{c}{ CTEQ-DIS } & EHLQ-DLA \\
& analytic & LS & LS \\
\hline ATM [92] & 4 & 3 & 3 \\
AGN-SS [97] & $62-75$ & $43-51$ & $39-46$ \\
AGN-NMB [99] & $42-49$ & $30-34$ & $27-31$ \\
AGN-SP [100] & $1060-1200$ & $747-843$ & $683-760$ \\
\hline
\end{tabular}

tributions for $E_{\mu}^{\min }=1 \mathrm{TeV}$. Consequently we only show rates corresponding to the EHLQ and CTEQ-DIS cross sections.

In Table 4 we show the upward-muon event rates for a detector with an effective area of $0.1 \mathrm{~km}^{2}$ and a muon energy threshold of $1 \mathrm{TeV}$. These event rates are for muons and antimuons with modern (CTEQ-DIS) and ancient (EHLQ-DLA) parton distribution functions, and show the difference between the analytic and Lipari-Stanev muon ranges. As a practical matter, we have taken the upper limit of the energy integral (40) to be $E_{\nu}^{\max }=10^{8} \mathrm{GeV}$, the limit of the Lipari-Stanev analysis of the muon range. The event rates from atmospheric neutrinos are roughly comparable to the AGN neutrino event rates for this muon energy threshold. In fact, most of the AGN event rate comes from the first few energy decades. The ATM rate comes entirely from $E_{\nu}<10^{6} \mathrm{GeV}$. For the AGN-SS flux, only about $75 \%$ of the rate comes from $E_{\nu}<10^{6} \mathrm{GeV}$, but by $E_{\nu}=10^{7} \mathrm{GeV}$, one has essentially all of the rate. About $5 \%$ of the AGN-NMB rate comes from neutrino energies above $10^{6} \mathrm{GeV}$. The details of the turnover of the AGN-NMB energy spectrum should not affect the predicted event rate significantly. The rates calculated with the CTEQ- 
DLA and D_ distributions give numerical rates essentially indistinguishable from the CTEQ-DIS numbers, as one would expect from Figure 22.

Table 5 shows the upward $\mu^{+}+\mu^{-}$event rate for a muon energy threshold of $10 \mathrm{TeV}$. The atmospheric neutrino background is significantly reduced. About $85 \%$ of the AGN-NMB rate arises from neutrino energies below $10^{6} \mathrm{GeV}$, an indication that the expected steepening of the spectrum may reduce the event rates reported in the table by some 10 to $20 \%$. In the AGN-SS model, for which the spectrum is predicted beyond $E_{\nu}=10^{9} \mathrm{GeV}$, more than $95 \%$ of the rate comes from $E_{\nu}<10^{7} \mathrm{GeV}$. Integrated over $2 \pi$ solid angle, the annual rates are very encouraging. We expect at least 190 AGN events on a ten-percent background. The atmospheric-muon background is negligible, except at the Earth's surface, where horizontal muons must be avoided. If the Szabo-Protheroe fluxes are correct, contrary to the Fréjus evidence [101], the detection of diffuse astrophysical neutrinos is imminent.

The cosmic-neutrino fluxes shown in Figure 18 are of interest for $E_{\nu}>10^{7} \mathrm{GeV}$. To evaluate the event rate for cosmic-neutrino interactions, we have evaluated the energy integrals from $10^{7} \mathrm{GeV}$ to $10^{12} \mathrm{GeV}$ using the analytic formula for the muon range. The upward event rates for muons with energies above $10^{7} \mathrm{GeV}$ are shown for a variety of parton distributions and detector conditions in Table 6. The CTEQ-DIS cross sections yield upward rates only about $20 \%$ larger than those implied by the EHLQ-DLA cross sections. The upward muon event rate appears to be very difficult to observe in a $0.1-\mathrm{km}^{2}$ detector.

To further explore the possibility of detecting cosmic neutrinos, we turn our attention to the downward and horizontal $\nu_{\mu} N$ event rates. The passage of neutrinos through the Earth reduces the upward angle-averaged neutrino flux by a factor of ten at $E_{\nu}=10^{7} \mathrm{GeV}$, and even further as the neutrino energy increases. The cosmic neutrino energy spectrum is nearly flat for $E_{\nu}$ between $10^{7} \mathrm{GeV} \lesssim E_{\nu} \lesssim 10^{9} \mathrm{GeV}$, so that in the absence of shadowing, the rate would be dominated by neutrinos with energies near the upper end of that range.

It is a good approximation to set the shadow factor to unity when considering downward neutrinos and for incident angles such that the column depth $z$ of the intervening rock is small compared to the neutrino interaction length. The range of interaction lengths for $E_{\nu}=10^{7}-10^{9} \mathrm{GeV}$ is $6.6 \times 10^{3}-9.4 \times$ $10^{2}$ kmwe. For a detector at the surface of the Earth, these lengths correspond to angles between $1.3^{\circ}$ and $8.9^{\circ}$ below horizontal. The detectors of interest are kilometers underground, so the precise angle at which the column depth equals the interaction length depends on details of the location of the detector. However, the numbers indicate in general that one can reliably set $S\left(E_{\nu}\right)=1$ only for neutrinos that are entering the detector from above or horizontally.

The downward event rates in Table 6 are calculated with no shadowing. Two 
sets of downward rates are shown: the first is for contained events in an effective volume of $V_{\text {eff }}=A \cdot 1 \mathrm{~km}=0.1 \mathrm{~km}^{3}$ for $E_{\nu}$ between $10^{7}$ and $10^{12} \mathrm{GeV}$, while the second set corresponds to $V_{\text {eff }}=A\langle R\rangle$, with $E_{\mu}^{\min }=10^{7} \mathrm{GeV}$.

For the contained events, the downward muon event rate is enhanced relative to the upward rate by a factor of 3 to 4 for the CTEQ-DIS parton distribution functions. Differences in parton distribution functions are much more striking in the downward event rate than for the upward event rate. In this case, the CTEQ-DIS rates are about twice as large as the old EHLQ-DLA rates. Even with the most optimistic flux and the highest (D_) estimate of the neutrinonucleon cross section, the contained rates for cosmic-neutrino interactions are very low.

A larger rate of muons from cosmic neutrinos would obtain if it were feasible to take advantage of the average muon range of about $10 \mathrm{~km}$. The second set of downward event rates uses the analytic range to establish the effective volume. The location of the detector will limit the range enhancement of the effective volume, since none of the planned detectors will be deployed at a depth of $10 \mathrm{~km}$. Even if one could take advantage of the full range enhancement over $2 \pi$ solid angle, the predicted rate for the CR-4 flux using the D_cross section is on the order of 0.3 event per year in a detector with $A=0.1 \mathrm{~km}^{2}$.

In our discussion of the downward event rates, we have not addressed the problem of the atmospheric muon background. For uncontained events, the neutrinos must interact to produce a muon signal, while the muons produced in the atmosphere by cosmic rays need only pass through the detector volume to be recorded. At $E_{\mu}=10^{7} \mathrm{GeV}$, the flux of muons is comparable to the flux of neutrinos in the CR-4 model at the Earth's surface [94]. Underground, the muon energy is degraded according to the range formula (37). To a good approximation, the muon flux is decreased by a factor of $\exp (-b \gamma z)$ when $d N / d E_{\mu} \propto E_{\mu}^{-(\gamma+1)}$. The vertical muon flux of (29) corresponds to $\gamma=2.7$. Taking $b=3.9 \times 10^{-6} \mathrm{cmwe}^{-1}$ as before, we find that the energy spectrum of the atmospheric muons below ground is degraded by a factor of about $\exp \left(-1.1 z \mathrm{kmwe}^{-1}\right)$. At a column depth of $8 \mathrm{kmwe}$, the suppression amounts to a factor of $\sim 10^{-4}$. Since the neutrino-to-muon conversion rate involves the multiplicative factor $N_{A} \sigma_{\mathrm{CC}}\left(E_{\nu}\right) L \approx 10^{-4}\left(E_{\nu} / 10^{7} \mathrm{GeV}\right)^{0.4}\left(L \mathrm{kmwe}^{-1}\right)$, the background from atmospheric muons is a concern at depths substantially less than 8 kmwe. To compensate, the solid angle must be restricted to include only large column depths. Consequently, it is overly optimistic to assume that uncontained neutrino-induced events can be observed over a $2 \pi$ solid angle. 
Table 6

The $\mu^{-}+\mu^{+}$event rates per steradian per year corresponding to two models of the cosmic neutrino flux (CR-2 and CR-4 [103]), for a detector with effective area $A=0.1 \mathrm{~km}^{2}$ and muon energy threshold $E_{\mu}^{\mathrm{min}}=10^{7} \mathrm{GeV}$. For upward events, we calculate the attenuation using the total cross section. For downward events we set $S\left(E_{\nu}\right)=1$.

\begin{tabular}{ccccc}
\hline Effective Volume & direction & parton distributions & CR-2 & CR-4 \\
\hline$A \cdot\langle R\rangle$ & upward & CTEQ-DIS & $1.9 \times 10^{-5}$ & $1.1 \times 10^{-3}$ \\
$A \cdot\langle R\rangle$ & upward & CTEQ-DLA & $1.8 \times 10^{-5}$ & $1.0 \times 10^{-3}$ \\
$A \cdot\langle R\rangle$ & upward & D_ & $1.9 \times 10^{-5}$ & $1.1 \times 10^{-3}$ \\
$A \cdot\langle R\rangle$ & upward & EHLQ-DLA & $1.6 \times 10^{-5}$ & $9.2 \times 10^{-4}$ \\
& & & & \\
$A \cdot 1 \mathrm{~km}$ & downward & CTEQ-DIS & $7.4 \times 10^{-5}$ & $3.5 \times 10^{-3}$ \\
$A \cdot 1 \mathrm{~km}$ & downward & CTEQ-DLA & $5.1 \times 10^{-5}$ & $2.6 \times 10^{-3}$ \\
$A \cdot 1 \mathrm{~km}$ & downward & D_ & $1.2 \times 10^{-4}$ & $4.9 \times 10^{-3}$ \\
$A \cdot 1 \mathrm{~km}$ & downward & EHLQ-DLA & $3.4 \times 10^{-5}$ & $1.8 \times 10^{-3}$ \\
$A \cdot\langle R\rangle$ & downward & CTEQ-DIS & $1.0 \times 10^{-3}$ & $3.3 \times 10^{-2}$ \\
$A \cdot\langle R\rangle$ & downward & CTEQ-DLA & $7.1 \times 10^{-4}$ & $2.5 \times 10^{-2}$ \\
$A \cdot\langle R\rangle$ & downward & D_ & $1.7 \times 10^{-3}$ & $4.8 \times 10^{-2}$ \\
$A \cdot\langle R\rangle$ & downward & EHLQ-DLA & $4.7 \times 10^{-4}$ & $1.7 \times 10^{-2}$ \\
\hline
\end{tabular}

\section{$8.3 \nu_{e}$ and $\bar{\nu}_{e}$ Interactions}

Finally we turn to the calculation of event rates involving electron neutrinos. Calculations for $\nu_{e} N$ charged-current interaction event rates proceed as above with $\nu_{\mu}$, except that the electron range is significantly shorter than the muon range. In general, only contained events can be observed because of the rapid energy loss (or annihilation) of electrons and positrons. Accordingly, event rates for electron neutrinos are smaller than muon event rates by the flux ratio times the detector length divided by the mean muon range. However, the rapid development of electromagnetic showers may make it possible to detect upward-going air showers initiated by an electron neutrino that interacts near the surface of the Earth. The Landau-Pomeranchuk-Migdal effect $[108,109]$ enhances the distance an electron can travel in the Earth. For electrons produced in $\nu_{e} N$ interactions at energy $E_{\nu}$, the mean path length is

$$
L_{\mathrm{LPM}}\left(E_{\nu}\right) \approx 40 \text { cmwe }\left[\left(1-\left\langle y\left(E_{\nu}\right)\right\rangle\right) \frac{E_{\nu}}{62 \mathrm{TeV}}\right]^{1 / 2}
$$


Table 7

Downward resonant $\bar{\nu}_{e} \rightarrow W^{-}$events per steradian per year for a detector with effective volume $V_{\text {eff }}=1 \mathrm{~km}^{3}$. Also shown are the potential downward (upward) background rates from $\nu_{\mu} N$ and $\bar{\nu}_{\mu} N$ interactions above $3 \mathrm{PeV}$.

\begin{tabular}{ccccc}
\hline Flux & $\bar{\nu}_{e} e \rightarrow \bar{\nu}_{\mu} \mu$ & $\bar{\nu}_{e} e \rightarrow$ hadrons & $\left(\nu_{\mu}, \bar{\nu}_{\mu}\right) N$ CC & $\left(\nu_{\mu}, \bar{\nu}_{\mu}\right) N$ NC \\
\hline AGN-SS [97] & 6 & 41 & $33(7)$ & $13(3)$ \\
AGN-SP [100] & 3 & 19 & $19(4)$ & $7(1)$ \\
\hline
\end{tabular}

A very-large-area air shower array might therefore constitute a large-volume detector for electron neutrinos.

Prospects for the detection of electron antineutrinos are more favorable around 6.3 PeV, the energy for resonant $W^{-}$formation in $\bar{\nu}_{e} e$ collisions. The contained event rate for resonant $W$ production is

$$
\text { Rate }=\frac{10}{18} N_{A} V_{\text {eff }} \int_{\left(M_{W}-2 \Gamma_{W}\right)^{2} / 2 m}^{\left(M_{W}+2 \Gamma_{W}\right)^{2} / 2 m} d E_{\bar{\nu}_{e}} \sigma_{\bar{\nu}_{e} e}\left(E_{\bar{\nu}_{e}}\right) \frac{d N_{\bar{\nu}_{e}}}{d E_{\bar{\nu}_{e}}} .
$$

We show in Table 7 the number of resonant $\bar{\nu}_{e} e$ events produced per steradian per year in a $1-\mathrm{km}^{3}$ detector for two models of the diffuse neutrino flux from AGNs that apply in this energy regime. We recall that, at the resonance energy, upward-moving electron antineutrinos do not survive passage through the Earth. The form $\propto(1-y)^{2}$ of the differential cross section (20) for $\bar{\nu}_{e} e \rightarrow \bar{\nu}_{\mu} \mu^{-}$ means that the mean energy of muons arising from $W^{-}$formation and decay will be $\left\langle E_{\mu}\right\rangle \approx \frac{1}{4} E_{\nu}^{\text {res }} \approx 1.4 \mathrm{PeV}$. The resonance signal is not background-free. We have also gathered in Table 7 the downward and upward rates for the charged-current $\left(\nu_{\mu} N \rightarrow \mu^{-}+\right.$anything and $\bar{\nu}_{\mu} N \rightarrow \mu^{+}+$anything) background to the $\bar{\nu}_{e} e \rightarrow W^{-} \rightarrow \bar{\nu}_{\mu} \mu^{-}$signal, and the downward and upward rates for the neutral-current $\left(\nu_{\mu} N \rightarrow \nu_{\mu}+\right.$ anything and $\bar{\nu}_{\mu} N \rightarrow \bar{\nu}_{\mu}+$ anything) background to the $\bar{\nu}_{e} e \rightarrow W^{-} \rightarrow$ hadrons signal. For this background estimate we have included all events induced by neutrinos with energies above $3 \mathrm{PeV}$. At the surface of the Earth, (29) leads to an estimate of 5 atmospheric-muon events per steradian per year above $3 \mathrm{PeV}$. Better discrimination against background is clearly desirable.

\section{Summary and Outlook}

We have studied the implications of new knowledge of nucleon structure at small values of $x$ for the detection of ultrahigh-energy neutrinos from extraterrestrial sources. Using a variety of modern parton distributions, we have calculated cross sections for the charged-current reactions, $\nu_{\mu} N \rightarrow \mu^{-}+$anything 
and $\bar{\nu}_{\mu} N \rightarrow \mu^{+}+$anything, that will be used to detect UHE neutrinos. Up to energies of about $10^{16} \mathrm{eV}$, parton distributions that entail different behaviors as $x \rightarrow 0$ yield very similar cross sections. The calculated cross sections are in good agreement with the charged-current cross section inferred from $e^{-} p$ interactions at HERA at an equivalent neutrino energy of $4.7 \times 10^{13} \mathrm{eV}$. At energies below $10^{15} \mathrm{eV}$, the new cross sections are about $15 \%$ larger than those calculated by Quigg, Reno, and Walker using the EHLQ structure functions and the double logarithmic approximation for the approach to $x=0$. At higher energies, the difference between new and old cross sections increases rapidly, reflecting the HERA observation of large parton densities at small $x$. At $10^{20} \mathrm{eV}$, our nominal cross sections, calculated from the CTEQ3 parton distributions, are about 2.4 times the EHLQ-DLA cross sections of a decade ago. In the regime above $10^{16} \mathrm{eV}$, the cross sections are sensitive to parton distributions at very small values of $x$, where there are no direct experimental constraints. Accordingly, different assumptions about the $x \rightarrow 0$ behavior lead to different cross sections. At $10^{20} \mathrm{eV}$, the resulting uncertainty reaches a factor of $2^{ \pm 1}$. We have also calculated the neutral-current $\nu_{\mu} N \rightarrow \nu_{\mu}+$ anything and $\bar{\nu}_{\mu} N \rightarrow \bar{\nu}_{\mu}+$ anything cross sections that contribute to the attenuation of UHE neutrinos as they traverse the Earth.

We have estimated event rates in large-volume detectors for downward- and upward-moving muons produced in charged-current interactions. The increased charged-current cross section translates directly into increased downward event rates, but the observation of downward events is complicated by the background of cosmic-ray muons. For upward events, the increased interaction rate is nearly compensated by the increased attenuation of UHE neutrinos in the Earth.

We expect that the new generation of neutrino telescopes will detect UHE neutrinos from extraterrestrial sources, and will begin to test models for neutrino production in active galactic nuclei. For the CTEQ-DIS cross sections and the Lipari-Stanev muon range-energy relation, we find that in one steradian-year, a detector with an active range of $0.02 \mathrm{~km}^{2}$ would record between 16 and 592 upward-moving muons with energies above $1 \mathrm{TeV}$ produced by interactions of AGN neutrinos, on a background of about 28 events produced by atmospheric neutrinos. If the muon energy threshold is raised to $10 \mathrm{TeV}$, the rates induced by diffuse AGN neutrinos will be between 9 and 170 events on a background of less than one event. The range of signal events reflects the spread in predictions of the diffuse neutrino flux from AGNs.

The outlook for the detection of cosmic neutrinos at energies around $10^{17} \mathrm{eV}$ is less encouraging. Even in a detector with an effective volume of $1 \mathrm{~km}^{3}$, the most favorable model for the cosmic-neutrino flux leads to less than one event per steradian-year with $E_{\mu}>10^{16} \mathrm{eV}$. 
Finally, we have considered the reaction $\bar{\nu}_{e} e \rightarrow W^{-}$as a means of probing the $\bar{\nu}_{e}$ spectrum in the neighborhood of the resonant energy, $6.3 \times 10^{15} \mathrm{eV}$. We estimate that a detector with effective volume $0.2 \mathrm{~km}^{3}$ would record between 4 and 7 downward $\bar{\nu}_{e} e \rightarrow W^{-} \rightarrow \bar{\nu}_{\mu} \mu$ events and between 24 and 50 downward hadronic events per year. The backgrounds from deeply inelastic $\nu_{\mu} N$ scattering are not negligible.

We are optimistic that progress toward large-volume neutrino telescopes, initially based on water-Čerenkov and ice-Čerenkov techniques, will soon lead to the detection of ultrahigh-energy neutrinos from extraterrestrial sources. With the ability to detect UHE neutrinos will come the possibility of looking deep within some of the most energetic structures in the universe. For neutrino energies up to $10^{16} \mathrm{eV}$, which spans the range of interest for testing models of active galactic nuclei, the neutrino-nucleon cross sections can be predicted with confidence. We expect neutrino telescopes to emerge as an important astrophysical tool.

\section{Acknowledgements}

We thank K. Daum, T. Gaisser, F. Halzen, A. Mann, M. Salamon, G. Smoot, T. Stanev, and F. Stecker for advice and encouragement.

Fermilab is operated by Universities Research Association, Inc., under contract DE-AC02-76CHO3000 with the United States Department of Energy. CQ thanks the Department of Physics and Laboratory of Nuclear Studies at Cornell University for warm hospitality. The research of MHR at the University of Iowa is supported in part by National Science Foundation Grant PHY 93-07213 and PHY 95-07688. The research of IS at the University of Arizona is supported in part by the United States Department of Energy under contracts DE-FG02-85ER40213 and DE-FG03-93ER40792. CQ, MHR, and IS acknowledge the hospitality of the Aspen Center for Physics.

\section{References}

[1] Y. Totsuka, Rep. Prog. Phys. 55 (1992) 377.

[2] T. K. Gaisser, F. Halzen, and T. Stanev, Phys. Rep. 258 (1995) 173.

[3] J. N. Bahcall, et al., Nature (London) 375 (1995) 29.

[4] S. Barwick, F. Halzen, and P. B. Price, "The Search for Neutrino Sources beyond the Sun," University of Wisconsin preprint MADPH-95-915 (electronic archive: astro-ph/9512079). 
[5] K. Hirata, et al. (KAMIOKANDE Collaboration), Phys. Rev. Lett. 58 (1987) 1490; R. M. Bionta, et al. (IMB Collaboration), ibid. 58 (1987) 1494.

[6] B. T. Cleveland, et al., in Neutrino 94, Proceedings of the Sixteenth International Conference on Neutrino Physics and Astrophysics, edited by A. Dar, G. Eilam, and M. Gronau Nucl. Phys. B (Proc. Supp.) 38 (1995) 47.

[7] P. Anselmann, et al. (GALLEX Collaboration), Phys. Lett. B285 (1992) 376; ibid. B314 (1993) 445; ibid. B327 (1994) 377; ibid. B342 (1995) 440.

[8] A. I. Abazov, et al. (SAGE Collaboration), Phys. Rev. Lett. 67 (1991) 3332; J. N. Abdurashitov, et al., Phys. Lett. B328 (1994) 234.

[9] K. Hirata, et al. (KAMIOKANDE Collaboration), Phys. Rev. D44 (1991) 2241.

[10] C. V. Achar, et al., Phys. Lett. 18 (1965) 196, ibid. 19 (1965) 78.

[11] F. Reines, et al., Phys. Rev. Lett. 15 (1965) 429.

[12] K. Daum, et al. (Fréjus Collaboration), Z. Phys. C66 (1995) 417. This paper contains extensive references to other modern experiments. See also F. Ronga, "High Energy Neutrinos in the MACRO Experiment," in Neutrino Telescopes, Proceedings of the Fifth International Workshop, Venice, 2-4 March 1993, edited by M. Baldo Ceolin, (University of Padua, Padua, 1993), p. 285.

[13] K. S. Hirata, et al. (KAMIOKANDE Collaboration), Phys. Lett. B280 (1992) 146.

[14] R. Becker-Szendy, et al. (IMB Collaboration), Phys. Rev. D46 (1992) 3720; and "Neutrino Measurements with the IMB Detector," in Neutrino 94, Proceedings of the Sixteenth International Conference on Neutrino Physics and Astrophysics, edited by A. Dar, G. Eilam, and M. Gronau Nucl. Phys. B (Proc. Supp.) 38 (1995) 331.

[15] M. C. Goodman, "The atmospheric neutrino anomaly in Soudan 2," in Neutrino 94, Proceedings of the Sixteenth International Conference on Neutrino Physics and Astrophysics, edited by A. Dar, G. Eilam, and M. Gronau Nucl. Phys. B (Proc. Supp.) 38 (1995) 337.

[16] V. S. Berezinsky, in TAUP 91, Proceedings of the 2nd International Workshop on Theoretical and Phenomenological Aspects of Underground Physics, Toledo, Spain, 9-13 September 1991, edited by A. Morales, J. Morales, and J.A. Villar, Nucl. Phys. B (Proc. Supp.) 28A (1992) 352.

[17] Todor Stanev, in Arkansas Gamma-Ray and Neutrino Workshop - 1989, Proceedings of the International Workshop on the Physics and Experimental Techniques of High Energy Neutrino and VHE and UHE Gamma-ray Particle Astrophysics, University of Arkansas, Little Rock, 10-13 May 1989, edited by Gaurang B. Yodh, Donald C. Wold, and William R. Krapp, Nucl. Phys. B (Proc. Supp.) 14A (1990) 17. 
[18] K. Greisen, Phys. Rev. Lett. 16 (1966) 748; V. Berezinsky and G. Zatsepin, in Proceedings of the 1976 DUMAND Summer Workshop, edited by A. Roberts and R. Donaldson (Fermilab, Batavia, Illinois, 1977), p. 215; C. T. Hill and D. N. Schramm, Phys. Rev. D31 (1985) 564; For a thorough modern treatment and extensive references to earlier work, see S. Yoshida and M. Teshima, Prog. Theoret. Phys. (Kyoto) 89 (1993) 833.

[19] The superconducting-cosmic-string scenario for galaxy formation was elaborated by J. P. Ostriker, C. Thompson, and E. Witten, Phys. Lett. B180 (1986) 231. The neutrino flux from the decay of superconducting cosmic strings was calculated by C. T. Hill, D. N. Schramm, and T. P. Walker, Phys. Rev. D36 (1987) 1007.

[20] Neutrino emission by ordinary cosmic strings has been treated, for example, by Jane H. MacGibbon and Robert H. Brandenburger, Nucl. Phys. B331 (1990) 153.

[21] P. Bhattacharjee, C. T. Hill, and D. N. Schramm, Phys. Rev. Lett. 69 (1992) 567.

[22] See, for example, V. L. Ginzburg and S. I. Syrovatskilu, The Origin of Cosmic Rays (Pergamon Press, Oxford, 1964); A. M. Hillas, Annu. Rev. Astron. Astrophys. 22 (1984) 425.

[23] See, for example, D. Eichler, Astrophys. J. 232 (1979) 106; M. Sikora, J. G. Kirk, M. C. Begelman, and P. Schneider, ibid. 320 (1987) L81.

[24] C. E. Fichtel, et al., Astrophys. J. Suppl. 94 (1994) 551.

[25] M. Punch, et al. (Whipple Observatory Gamma Ray Collaboration), Nature (London) 160 (1992) 477; A. D. Kerrick, et al., Astrophys. J. 438 (1995) L59. The nominal distance to Mrk 421 is 400 million light years.

[26] J. Quinn, et al. (Whipple Observatory Gamma Ray Collaboration), IAU Circular 6169 (June 16, 1995).

[27] For a general discussion, see T. K. Gaisser, Cosmic Rays and Particle Physics, (Cambridge University Press, Cambridge, 1990).

[28] For a survey of detectors for neutrino astronomy, see J. G. Learned, Phil. Trans. Roy. Soc. London A 346 (1994) 99.

[29] The prototype water Čerenkov detectors are the underground KAMIOKANDE and IMB detectors cited above. The Super-Kamiokande Project is described by K. Nakamura, in NESTOR, Third NESTOR International Workshop, 1921 October 1993, edited by L. K. Resvanis (Athens University Press, Athens, 1994), p. 151.

[30] The Deep Underwater Muon and Neutrino Detector is described by V. Z. Peterson, in Currents in Astrophysics and Cosmology: Papers in Honor of Maurice M. Shapiro, edited by G. G. Fazio and R. Silberberg (Cambridge University Press, Cambridge, 1994), p. 169; P. K. F. Grieder, in NESTOR, 
Third NESTOR International Workshop, 19-21 October 1993, edited by L. K. Resvanis (Athens University Press, Athens, 1994), p. 168. For a personal history of the DUMAND Project, see A. Roberts, Rev. Mod. Phys. 64 (1992) 259 .

[31] See also R. J. Wilkes, "DUMAND and AMANDA: High-Energy Neutrino Astrophysics," University of Washington preprint UWSEA-PUB-94-07 (electronic archive: astro-ph/9412019), to be published in the proceedings of 22nd Annual SLAC Summer Institute on Particle Physics: Particle Physics, Astrophysics and Cosmology, Stanford, CA, 8-19 August 1994.

[32] The Baikal Neutrino Telescope is described by R. Wischnewski, in NESTOR, Third NESTOR International Workshop, 19-21 October 1993, edited by L. K. Resvanis (Athens University Press, Athens, 1994), p. 213; R. Wischnewski, in Neutrino Telescopes, Proceedings of the Fifth International Workshop, Venice, 2-4 March 1993, edited by M. Baldo Ceolin, (University of Padua, Padua, 1993), p. 299; Ch. Spiering, in TAUP 91, Proceedings of the 2nd International Workshop on Theoretical and Phenomenological Aspects of Underground Physics, Toledo, Spain, 9-13 September 1991, edited by A. Morales, J. Morales, and J.A. Villar, Nucl. Phys. B (Proc. Supp.) 28A (1992) 491. See also J. Krabi, et al., "Sensitivity of the Baikal Neutrino Telescope NT-200 to Point Sources of Very High Energy Neutrinos," Institute for High Energy Physics, Zeuthen preprint PHE 91-13 (unpublished).

[33] A neutrino particle astrophysics underwater laboratory in the Mediterranean is described by L. K. Resvanis, in Neutrino Telescopes, Proceedings of the Fifth International Workshop, Venice, 2-4 March 1993, edited by M. Baldo Ceolin, (University of Padua, Padua, 1993), p. 321; L. K. Resvanis, in NESTOR, Third NESTOR International Workshop, 19-21 October 1993, edited by L. K. Resvanis (Athens University Press, Athens, 1994), p. 1.

[34] The Antarctic Muon and Neutrino Detector Array is described by R. Morse, in Neutrino Telescopes, Proceedings of the Fifth International Workshop, Venice, 2-4 March 1993, edited by M. Baldo Ceolin, (University of Padua, Padua, 1993), p. 309. For a recent report of the optical properties of the South Pole ice, see P. Askebjer, et al., Science 267 (1995) 1147.

[35] John P. Ralston and Douglas W. McKay, "ICEMAND: Microwave Detection of Ultrahigh-Energy Neutrinos in Ice," in Arkansas Gamma-Ray and Neutrino Workshop - 1989, Proceedings of the International Workshop on the Physics and Experimental Techniques of High Energy Neutrino and VHE and UHE Gamma-ray Particle Astrophysics, University of Arkansas, Little Rock, 1013 May 1989, edited by Gaurang B. Yodh, Donald C. Wold, and William R. Krapp, Nucl. Phys. B (Proc. Supp.) 14A (1990) 356. G. M. Frichter, J. P. Ralston, and D. W. McKay, "On Radio Detection of Ultrahigh-Energy Neutrinos," Kansas University preprint KITCS-95-1-3 (electronic archive: astro-ph/9507078). A. L. Provorov and I. M. Zheleznykh, Astropart. Phys. 4 (1995) 55.

[36] T. Bowen, Sixteenth International Cosmic Ray Conference, vol. 2, p. 184 (University of Kyoto, 1979). 
[37] C. Quigg, M. H. Reno, and T. P. Walker, Phys. Rev. Lett. 57 (1986) 774.

[38] M. H. Reno and C. Quigg, Phys. Rev. D37 (1988) 657.

[39] D. W. McKay and J. P. Ralston, Phys. Lett. 167B (1986) 103.

[40] A. V. Butkevich, A. B. Kaŭdalov, P. I. Krastev, A. V. Leonov-Vendrovski, and I. M. Zheleznykh, Z. Phys. C39 (1988) 241.

[41] E. Eichten, I. Hinchliffe, K. Lane, and C. Quigg, Rev. Mod. Phys. 56 (1984) 579; ibid. 58 (1986) 1065E.

[42] F. Abe, et al. (CDF Collaboration), Phys. Rev. Lett. 74 (1995) 2626.

[43] S. Abachi, et al. (DØ Collaboration), Phys. Rev. Lett. 74 (1995) 2632.

[44] The first to examine the implications of HERA data for the UHE neutrino cross section were George M. Frichter, Douglas W. McKay, and John P. Ralston, Phys. Rev. Lett. 74 (1995) 1508. These authors used HERA data to parametrize the low- $x$ parton distributions, under the Ansatz $q(x)=$ $\bar{q}(x)=u(x)=d(x)=s(x)=2 c(x)=2 b(x)$, for which $F_{2}^{e p}=\frac{17}{9} x q(x)$ and $\mathcal{F}_{2}^{\nu N}=8 x q(x)$.

[45] M. Derrick, et al. (ZEUS Collaboration), Phys. Lett. B316 (1993) 412.

[46] I. Abt, et al. (H1 Collaboration), Nucl. Phys. B407 (1993) 515.

[47] G. Wolf, "HERA Physics," in High Energy Phenomenology, Proceedings of the 42nd Scottish Universities Summer School in Physics, St. Andrews, 1-21 August 1993, edited by K. J. Peach and L. L. J. Vick (SUSSP Publications, Edinburgh, and Institute of Physics Publishing, London, 1994), p. 135.

[48] M. Derrick, et al. (ZEUS Collaboration), Z. Phys. C65 (1995) 379.

[49] T. Ahmed, et al. (H1 Collaboration), Nucl. Phys. B439 (1995) 471.

[50] G. Altarelli and G. Parisi, Nucl. Phys. B126 (1977) 298. See also V. N. Gribov and L. N. Lipatov, Yad. Fiz. 15 (1972) 781 [English transl.: Sov. J. Nucl. Phys. 15 (1972) 438]; L. N. Lipatov, Yad. Fiz. 20 (1974) 181 [English transl.: Sov. J. Nucl. Phys. 20 (1974) 94]; Yu. L. Dokshitser, Zh. Eksp. Teor. Fiz. 73 (1977) 1216 [English translation: Sov. Phys.-JETP 46 (1977) 641].

[51] H. Lai, et al. (CTEQ Collaboration), Phys. Rev. D51 (1995) 4763.

[52] A. D. Martin, W. J. Stirling, and R. G. Roberts, Phys. Rev. D50 (1994) 6734.

[53] A. D. Martin, W. J. Stirling, and R. G. Roberts, Phys. Lett. B354 (1995) 155.

[54] A. D. Martin, W. J. Stirling, and R. G. Roberts, Phys. Rev. D47 (1993) 867.

[55] A. D. Martin, W. J. Stirling, and R. G. Roberts, Phys. Lett. B306 (1993) 145. 
[56] E. A. Kuraev, L. N. Lipatov, and V. S. Fadin, Zh. Eksp. Teor. Fiz. 71 (1976) 840 [English translation: Sov. Phys.-JETP 44 (1976) 443]; Zh. Eksp. Teor. Fiz. 72 (1977) 377 [English translation: Sov. Phys.-JETP 45 (1977) 199]; Ya. Ya. Balitskiǔ, and L. N. Lipatov, Yad. Fiz. 28 (1978) 1597 [English transl.: Sov. J. Nucl. Phys. 28 (1978) 822]; L. N. Lipatov, Zh. Eksp. Teor. Fiz. 90 (1986) 1536 [English translation: Sov. Phys.-JETP 63 (1986) 904]; L. N. Lipatov, "Pomeron in Quantum Chromodynamics," in Perturbative Quantum Chromodynamics, edited by A. H. Mueller (World Scientific, Singapore, 1989), p. 411.

[57] G. Marchesini, "Structure Function for Large and Small $x$," in $Q C D$ at 200 $\mathrm{TeV}$, Proceedings of the 17th Workshop of the INFN ELOISATRON Project, Erice, 1991, edited by Luisa Cifarelli and Yuri Dokshitzer (Plenum, New York and London, 1992), p. 183.

[58] E. Laenen and E. Levin, Nucl. Phys. B451 (1995) 207.

[59] L. V. Gribov, E. M. Levin, and M. G. Ryskin, Phys. Rep. 100 (1983) 1.

[60] See also McKay and Ralston, Ref. [39].

[61] Eichten, et al., Ref. [41] did investigate singular starting distributions [Set 1(a)], but these have not been employed for UHE neutrino scattering.

[62] Recently, a detailed study of small- $x$ evolution, including the applicability of the DLA, has been carried out by R. K. Ellis, Z. Kunszt, and E. M. Levin, Nucl. Phys. B420 (1994) 517. One can conclude from their analysis that for the relevant range $\lambda \simeq 0.28-0.35$, the DLA form should be approximately correct for $x>10^{-5}$ and $Q^{2} \sim M_{W}^{2}$. Below $x \approx 10^{-5}$, the perturbative expansion of the Altarelli-Parisi splitting functions is unreliable. Higher order corrections to the splitting functions result in larger parton distribution functions at small $x$.

[63] It is demonstrated in Ref. [62], and in R. K. Ellis, F. Hautmann, and B. R. Webber, Phys. Lett. B348 (1995) 582, that for $Q^{2} \geq Q_{0}^{2}$, the small- $x$ extrapolation for $\lambda=0.5$ distributions is itself of the form $x q_{s}\left(x, Q^{2}\right) \sim$ $A(Q) x^{-0.5}$. We have evaluated the change in the power of $x$ with the numerical MRS D_ evolution, and found that $x q_{s}\left(x, Q^{2}\right)=A(Q) x^{-0.48}$ at $x=10^{-4}$ for $\sqrt{Q^{2}}$ between $50 \mathrm{GeV}$ and $150 \mathrm{GeV}$. This form is little changed as one goes to $x=10^{-5}$, the limit of the numerically evolved $\mathrm{D}_{\text {- }}$ parton distribution functions. We use this power law in our small- $x$ extrapolation of the MRS D_ set. The normalization constant $A(Q)$ is evaluated as a function of $Q$ at $x^{\mathrm{min}}$, so that the extrapolated form matches the numerically evolved sea-quark distributions.

[64] R. M. Barnett, Phys. Rev. D14 (1976) 70.

[65] See G. Altarelli, R. K. Ellis, and G. Martinelli, Nucl. Phys. B143 (1978) 521.

[66] G. Peter Lepage, J. Comput. Phys. 27 (1978) 192; Cornell University Report CLNS-80/447 (unpublished). 
[67] Yu. M. Andreev, V. S. Berezinsky, and A. Yu. Smirnov, Phys. Lett. 84B (1979) 247. See also V. S. Berezinskil and A. Z. Gazizov, Yad. Fiz. 29 (1979) 1589 [English transl.: Sov. J. Nucl. Phys. 29 (1979) 816].

[68] H. Abramowitz, et al. (CDHS Collaboration), Z. Phys. C17 (1983) 283.

[69] S. R. Mishra, et al. (CCFR Collaboration), Nevis Laboratory Report Nevis1465 (1992), and in Lepton-Hadron Scattering, Proceedings of the Nineteenth SLAC Summer Institute on Particle Physics, edited by Jane Hawthorne, SLAC-REPORT-398 (1992), p. 407.

[70] I. Abt, et al. (H1 Collaboration), Phys. Lett. B324 (1994) 241; S. Aid, et al. (H1 Collaboration), Z. Phys. C67 (1995) 565. The average charged-current cross section displayed in Figure 5 was presented by Rolf Beyer at the 1995 Workshop on Weak Interactions and Neutrinos, Talloires, France.

[71] The ZEUS Collaboration (M. Derrick, et al., Phys. Rev. Lett. 75 (1995) 1006) has published measurements of $d \sigma_{\mathrm{CC}}(\nu N) / d Q^{2}$ that are consistent with the MRS D_' distributions, which are equivalent, over the range of $x$ and $Q^{2}$ probed, to the distributions we consider here.

[72] G. Parente and E. Zas, "The High Energy Neutrino-Nucleon Cross Section from Recent HERA Parton Densities," Contribution EPS 0127 to the International Europhysics Conference on High Energy Physics (HEP 95), Brussels.

[73] A. V. Butkevich, et al., "Prospects for Radio Wave and Acoustic Detection of Ultra and Super High Energy Cosmic Neutrinos (Cross Sections, Signals, Thresholds)," Contribution EPS 0523 to the International Europhysics Conference on High Energy Physics (HEP 95), Brussels.

[74] M. Glück, E. Reya, and A. Vogt, Z. Phys. C53 (1992) 127.

[75] J. G. Morfin and W.-K. Tung, Z. Phys. C52 (1991) 13.

[76] Dorothée Schaile, "Precision Tests of the Electroweak Interaction," in Proceedings of the XXVII International Conference on High Energy Physics, edited by P. J. Bussey and I. G. Knowles (Institute of Physics Publishing, Bristol and Philadelphia, 1995), Volume I, p. 27.

[77] A thorough study of intermediate-boson production in ep collisions at HERA has been carried out by M. Böhm and A. Rosado, Z. Phys. C34 (1987) 117; ibid. 39 (1988) 275.

[78] V. S. Berezinskiǔ and A. Z. Gazizov, Yad. Fiz. 33 (1981) 230 [English transl.: Sov. J. Nucl. Phys. 33 (1981) 120], made a thorough study of neutrinoelectron scattering in the Weinberg-Salam theory, before the discovery of the electroweak gauge bosons. These authors also explore the effect of Fermi motion and radiative corrections.

[79] S. L. Glashow, Phys. Rev. 118 (1960) 316 suggested the reaction $\bar{\nu}_{e} e \rightarrow W^{-} \rightarrow$ $\bar{\nu}_{\mu} \mu^{-}$to look for evidence of a light $W$-boson in the interactions of atmospheric 
neutrinos. The important generalization to the reaction $\bar{\nu}_{e} e \rightarrow W^{-} \rightarrow$ hadrons was made by V. S. Berezinsky and A. Z. Gazizov, ZhETF Pis'ma 25 (1977) 276 [English translation: JETP Lett. 25 (1977) 254]. F. Wilczek, Phys. Rev. Lett. 55 (1985) 1252, emphasized the peculiar characteristics of charm showers in the reaction $\bar{\nu}_{e} e \rightarrow W^{-} \rightarrow \bar{c} s$.

[80] A compendium of $\bar{\nu}_{e} e$ reactions, including formation of hadronic resonances, is given by K. O. Mikaelian and I. M. Zheleznykh, Phys. Rev. D22 (1980) 2122. (They also treat the radiative correction to $W^{-}$formation.) For a recent update of resonance cross sections, see Myron Bander and H. R. Rubinstein, Phys. Rev. D51 (1995) 1410.

[81] Particle Data Group, Phys. Rev. D50 (1994) 1173.

[82] The use of seismic waves to infer the internal structure of the Earth is described in Bruce A. Bolt, Inside the Earth (W. H. Freeman, San Francisco, 1982), reprinted 1992 by Tech Books, Fairfax, Virginia, and in Bruce A. Bolt, Earthquakes and Geological Discovery (Scientific American Library, New York, 1993). See also Martin H. P. Bott, The Interior of the Earth (St. Martin's Press, New York, 1971).

[83] Parameters of the Preliminary Reference Earth Model are given by Adam Dziewonski, "Earth Structure, Global," in The Encyclopedia of Solid Earth Geophysics, edited by David E. James (Van Nostrand Reinhold, New York, 1989), p. 331.

[84] The US Standard Atmosphere (1976) is given, for example, in the CRC Handbook of Chemistry and Physics, 75th Edition, edited by David R. Lide (CRC Press, Boca Raton, Florida, 1994) p. 14-14.

[85] R. M. Baltrusaitis, et al., Nucl. Instrum. Methods Phys. Res., Sect. A 240 (1985) 410.

[86] F. Halzen, R. A. Vázquez, T. Stanev, and H. P. Vankov, Astropart. Phys. 3 (1995) 151.

[87] D. J. Bird, et al., Phys. Rev. Lett. 71 (1993) 3401. The estimated energy of the shower is $3.0_{-0.54}^{+0.36} \times 10^{20} \mathrm{eV}$.

[88] G. Sigl and S. Lee, Twenty-fourth International Cosmic Ray Conference, vol. 2, p. 356 (University of Rome, 1995).

[89] The original proposal to observe the shadow of the Moon and Sun in highenergy cosmic rays is due to G. W. Clark, Phys. Rev. 108 (1957) 450. The first observation of shadowing was made by D. E. Alexandreas, et al., Phys. Rev. $D 43$ (1991) 1735. See also M. Amenomori, et al. (Tibet AS $\gamma$ Collaboration), Phys. Rev. D47 (1993) 2675; A. Borione, et al., Phys. Rev. D49 (1994) 1171.

[90] See T. L. Wilson, "Medium and High-Energy Neutrino Physics from a Lunar Base," in Physics and Astrophysics from a Lunar Base, edited by A. E. Potter and T. L. Wilson, AIP Conference Proceedings 202 (American Institute of Physics, New York, 1990), p. 53. 
[91] J. N. Bahcall and R. K. Ulrich, Rev. Mod. Phys. 60 (1988) 297. The density profile is given in Table $\mathrm{X}$.

[92] L. V. Volkova, Yad. Fiz. 31 (1980) 1510 [English transl.: Sov. J. Nucl. Phys. 31 (1980) 784]. The angle-average fluxes are given in Tables 2 and 3.

[93] Other simulations of the atmospheric neutrino fluxes are in good agreement with the Volkova calculation in the range of interest to us: T. K. Gaisser, T. Stanev, and G. Barr, Phys. Rev. D38 (1988) 85; G. Barr, T. K. Gaisser, and T. Stanev, Phys. Rev. D39 (1989) 3532; V. Agrawal, T. K. Gaisser, P. Lipari, and T. Stanev, Bartol preprint BA-95-49 (electronic archive: hepph/9509423); A. Butkevich, L. G. Dedenko, and I. M. Zheleznyh, Yad. Fiz. 50 (1989) 142 [English transl.: Sov. J. Nucl. Phys. 50 (1989) 90]; K. Mitsui, Y. Minorikawa, and H. Komori, Nuovo Cim. 9C (1986) 995; P. Lipari, Astropart. Phys. 1 (1993) 195. For a comparison with data, see Figure 10 of Reference $[12]$.

[94] P. Gondolo, G. Ingelman, and M. Thunman, "Charm Production and High Energy Atmospheric Muon and Neutrino Fluxes," Uppsala preprint TSL/ISV95-0120, (electronic archive: hep-ph/9505417).

[95] For a compilation of measurements of the muon flux as a function of depth, see Figure 7 of L. K. Resvanis, first paper of Ref. [33]. The attenuation of cosmicray muons in matter closely follows the empirical formula given by S. Miyake, Thirteenth International Cosmic Ray Conference, vol. 5, p. 3638 (University of Denver, 1973).

[96] See the early calculations of neutrino fluxes from $\pi^{ \pm}$production in $p p$ collisions by V. S. Berezinsky and A. Yu. Smirnov, Ap. Space Sci. 32 (1975) 461; V. S. Berezinskiu and G. T. Zatsepin, Usp. Fiz. Nauk. 122 (1977) 3 [English translation: Sov. Phys. Usp. 20 (1977) 361].

[97] F. W. Stecker, C. Done, M. H. Salamon, and P. Sommers, Phys. Rev. Lett. 66 (1991) 2697; ibid. 69 (1992) 2738E. Revised estimates of the neutrino flux appear in F. W. Stecker and M. H. Salamon, "High Energy Neutrinos from Quasars," (electronic archive: astro-ph/9501064), submitted to Space Science Reviews.

[98] See V. S. Berezinskiǔ, "High Energy Neutrinos from Active Galactic Nuclei," in Neutrino 94, Proceedings of the 16th International Conference on Neutrino Physics and Astrophysics, Eilat, Israel, edited by A. Dar, G. Eilam, and M. Gronau, Nucl. Phys. B (Proc. Supp.) 38 (1995) 363, and $\S 7$ of Ref. [2] for reviews of several models. See also K. Mannheim, Astropart. Phys. 3 (1995) 295 and D. Kazanas, "Radio-Loud AGN As Sources of High-Energy Neutrinos," in NESTOR, Third NESTOR International Workshop, 19-21 October 1993, edited by L. K. Resvanis (Athens University Press, Athens, 1994), p. 29.

[99] L. Nellen, K. Mannheim, and P. L. Biermann, Phys. Rev. D47 (1993) 5270.

[100] A. P. Szabo and R. J. Protheroe, Astropart. Phys. 2 (1994) 375. 
[101] W. Rhode, et al. (Fréjus Collaboration), "Limits on the flux of very high energy neutrinos with the Fréjus Detector," Wuppertal preprint WUB-95-26, to appear in Astroparticle Physics. This paper contains extensive comparisons with models of the very-high-energy neutrino flux.

[102] M. Aglietta, et al. (EAS-TOP Collaboration), Phys. Lett. B333 (1994) 555.

[103] S. Yoshida and M. Teshima, Ref. [18].

[104] C. T. Hill and D. N. Schramm, Ref. [18]; V. S. Berezinsky and S. I. Grigor'eva, Astron. Astrophys. 199 (1988) 1.

[105] V. S. Berezinskiǔ, A. Z. Gazizov, G. T. Zatsepin, and I. L. Rozental, Yad. Fiz. 43 (1986) 637 [English transl.: Sov. J. Nucl. Phys. 43 (1986) 406].

[106] The energy-loss parameters are taken from p. 1256 of Reference [81].

[107] P. Lipari and T. Stanev, Phys. Rev. D44 (1991) 3543.

[108] L. D. Landau and I. Ya. Pomeranchuk, Dokl. Akad. Nauk SSSR 92, 535 (1953); ibid. 92 (1953) 735. English translations are contained in The Collected Papers of L. D. Landau, edited by D. Ter Haar (Pergamon Press \& Gordon and Breach, London, 1965), pp. 586, 589. A. B. Migdal, Phys. Rev. 103 (1956) 1811.

[109] A quantitative study of the LPM effect has been made by P. L. Anthony, et al., Phys. Rev. Lett. 75 (1995) 1949. 